\title{
Aeroservoelastic Wind-Tunnel Test of the SUGAR Truss Braced Wing Wind-Tunnel Model
}

\author{
Robert C. Scott, ${ }^{*}$ Timothy J. Allen, ${ }^{\dagger}$ Christie J. Funk, ${ }^{\ddagger}$ Mark A. Castelluccio,${ }^{\S}$ \\ Bradley W. Sexton, ${ }^{\uparrow}$ Scott Claggett,, John Dykman, ${ }^{* *}$ David A. Coulson, ${ }^{\dagger \dagger}$ \\ and Robert E. Bartels ${ }^{\ddagger \ddagger}$
}

\begin{abstract}
The Subsonic Ultra Green Aircraft Research (SUGAR) Truss-Braced Wing (TBW) aeroservoelastic (ASE) wind-tunnel test was conducted in the NASA Langley Transonic Dynamics Tunnel (TDT) and was completed in April, 2014. The primary goals of the test were to identify the open-loop flutter boundary and then demonstrate flutter suppression. A secondary goal was to demonstrate gust load alleviation (GLA). Open-loop flutter and limit cycle oscillation onset boundaries were identified for a range of Mach numbers and various angles of attack. Two sets of control laws were designed for the model and both sets of control laws were successful in suppressing flutter. Control laws optimized for GLA were not designed; however, the flutter suppression control laws were assessed using the TDT Airstream Oscillation System. This paper describes the experimental apparatus, procedures, and results of the TBW wind-tunnel test. Acquired system ID data used to generate ASE models is also discussed.
\end{abstract}

\section{Introduction}

The NASA Fixed Wing program has funded a number of research projects for the purpose of addressing multiple $\mathrm{N}+3$ goals, including reduced noise, emissions, and energy consumption. To reduce energy consumption, propulsive and aerodynamic efficiency increases have been addressed in recent research efforts including a Phase I study by The Boeing Company. ${ }^{1}$ A key outcome of this study was the conceptual design of the Subsonic Ultra Green Aircraft Research (SUGAR) Truss-Braced Wing (TBW) configuration shown in Figure 1. The configuration showed significant potential to contribute to meeting NASA N+3 goals, but also highlighted a significant uncertainty in the wing weight estimate.

One of the key objectives of the SUGAR Phase II study was to refine the TBW configuration and reduce the uncertainty in the potential benefits with specific focus on reducing the uncertainty of the wing weight. The study included the development of a detailed full scale, finite element model (FEM) of the wing and center body. This model was used to optimize the wing design subject to multiple design constraints including flutter. To validate the flutter predictions, an aeroelastically scaled wind-tunnel model was designed, fabricated, and tested. Other goals of the wind-tunnel test were to demonstrate flutter suppression and assess the control law effects on gust loads. The full scale and model scale FEMs along with the wind-tunnel model design are described in detail in References 2 and 3. The purpose of this paper is to summarize the wind-tunnel test and associated results.

The TBW wind-tunnel model was tested in the NASA Langley Transonic Dynamics Tunnel (TDT) from December 2013 to April 2014. Both open-loop and closed-loop data were acquired as part of this aeroservoelastic test, requiring the inclusion of high bandwidth ailerons in the design. Since the 1960s, the

\footnotetext{
*Senior Aerospace Engineer, Aeroelasticity Branch, NASA Langley Research Center, Hampton, VA., AIAA Associate Fellow.

${ }^{\dagger}$ Principal Engineer, Boeing Research \& Technology, Huntington Beach, CA.

$¥$ Aerospace Engineer, Aeroelasticity Branch, NASA Langley Research Center, Hampton, VA., AIAA Member.

$\S$ Guidance, Navigation \& Controls Engineer, The Boeing Company, Seattle, WA.

ฯ Principal Engineer, Boeing Research \& Technology, St. Louis, MO., AIAA Associate Fellow.

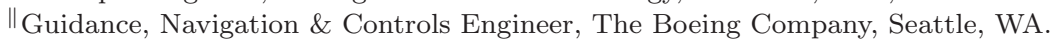

** Loads \& Aeroelasticity, Boeing Research \& Technology, Huntington Beach, CA., AIAA Member.

${ }^{\dagger}$ Senior Research Engineer, Analytical Services \& Materials, Inc., Hampton, VA.

${ }^{\ddagger}$ Senior Aerospace Engineer, Aeroelasticity Branch, NASA Langley Research Center, Hampton, VA., AIAA Senior Member.
} 


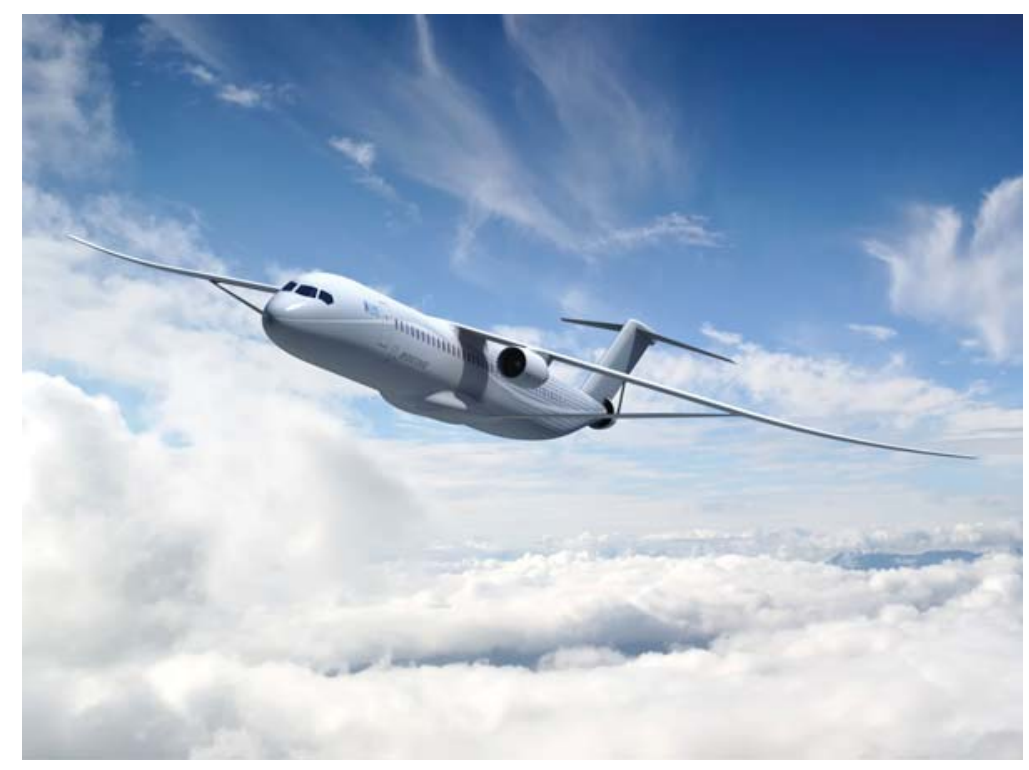

Figure 1. SUGAR TBW vehicle concept developed during the Phase I study.

TDT has conducted a variety of aeroservoelastic (ASE) wind-tunnel tests including the first wind-tunnel demonstration of flutter suppression on a large scale model. The history of TDT active-controls testing through 2000 is described in Reference 4 . Since that time, there have been significant improvements in both the hardware and associated software development environments for implementing and testing control laws. In addition, custom hardware, software, and procedures have been developed for use at the TDT in support of projects and programs such as Aerodynamic Efficiency Improvement (AEI) ${ }^{5,6}$ and the SemiSpan Supersonic Transport $\left(\mathrm{S}^{4} \mathrm{~T}\right) .{ }^{7}$ These systems were modified to support the TBW test and will be described in this paper.

The TBW wind-tunnel test results showed the flutter mechanism to primarily be a coalescence of $2^{\text {nd }}$ bending mode and $1^{\text {st }}$ torsion mode near $10 \mathrm{~Hz}$, as predicted by analysis. Results also showed a change in the flutter and limit cycle oscillation (LCO) boundary as angle of attack (AOA) was varied. This nonlinear behavior can at least partially be explained by including preload and large displacement changes to the structural stiffness and mass matrices in the flutter analysis as described in Reference 8. The LCO behavior was partially replicated with CFD and linear structural analysis as described in Reference 9. Control laws designed using ASE models (derived from experimental data and FEM models) were successful in suppressing flutter.

This paper will provide descriptions of all testing apparatus including the TDT, the TBW wind-tunnel model, and the digital control systems. An overview of the digital control system controller architecture will be presented. Open-loop testing procedures will be described and data presented in various forms. The ASE models used for control law design will be described. Finally closed-loop testing procedures and results will be described.

\section{Transonic Dynamics Tunnel and the Airstream Oscillation System}

The NASA Langley Transonic Dynamics Tunnel, depicted in Figure 2a, is a unique national facility dedicated to identifying, understanding, and solving relevant aeroelastic and aeroservoelastic problems. The TDT is a closed-circuit, continuous-flow, variable-pressure, wind tunnel with a 16-foot square test section with cropped corners. ${ }^{10}$ The tunnel uses either air or a heavy gas as the test medium and can operate at total pressures from near vacuum to atmospheric. It has a Mach number range from near zero to 1.2 and is capable of maximum Reynolds numbers of about 3 million per foot in air and 10 million per foot in heavy gas. Until 1996, the TDT used Dichlorodifluoromethane, R-12, as the heavy gas test medium; since then the TDT has used 1,1,1,2 Tetrafluoroethane, R-134a, ${ }^{11,12}$ an environmentally acceptable alternative to R-12. 
The TDT is specially configured for flutter testing, with excellent model visibility from the control room and a rapid tunnel shutdown capability (bypass valves) for model safety. Testing in heavy gas has important advantages over testing in air: improved model to full-scale similitude (which results in heavier, easier-tobuild models with lower elastic mode frequencies), higher Reynolds numbers, and reduced tunnel power requirements.

Due to the desire to perform GLA testing as part of the TBW wind-tunnel test, the Airstream Oscillation System (AOS) was also used. The key features of the AOS are shown in the illustration in Figure 2b. These features include biplane arrangements of vanes on either side of the entrance to the test section. Each pair of vanes is driven by a separate hydraulic motor and a flywheel to hold constant vane frequency. While the two pairs of vanes can be run out of phase, this was not done for the TBW test. Vane frequency was adjusted manually from the TDT control room. The amplitude of the vanes is manually adjustable from $0^{\circ}$ to $12^{\circ}$ peak-to-peak. The vane amplitude was set to $3^{\circ}$ peak-to-peak for the TBW wind-tunnel test.

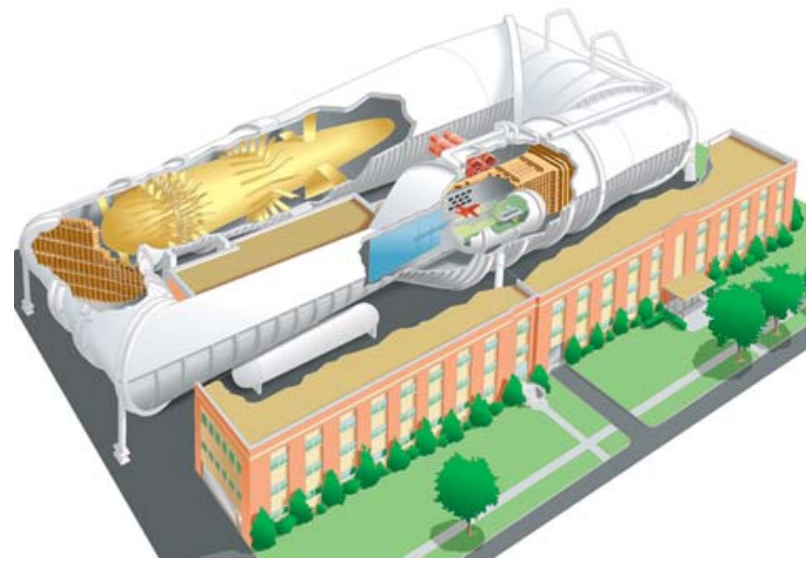

(a) TDT

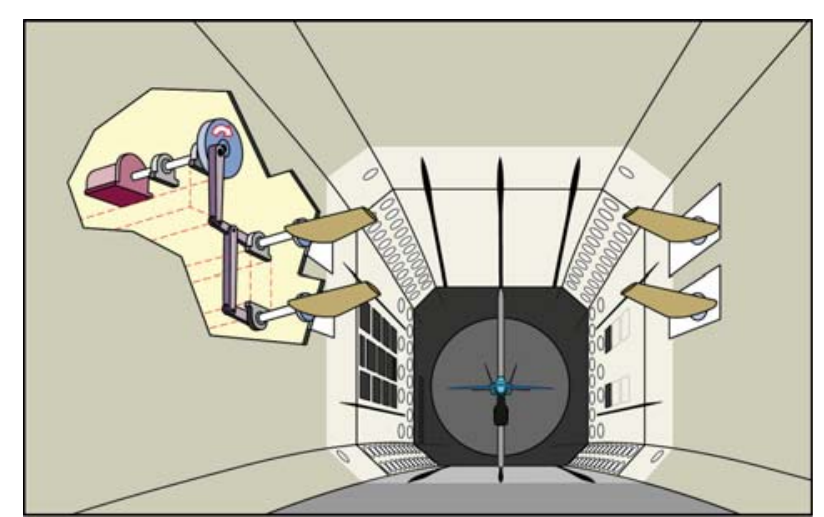

(b) AOS

Figure 2. Illustration of the NASA Langley Transonic Dynamics Tunnel (TDT) and associated Airstream Oscillation System (AOS) used to conduct the TBW test.

The TBW wind-tunnel model was designed for heavy gas testing so most of the experimental data were acquired in the R-134a test medium. Figure 3 shows the TDT heavy gas operating boundary illustrating Mach number $(\mathrm{M})$ and dynamic pressure $(\overline{\mathrm{q}})$ combinations that can be achieved in the test section. Operationally at the TDT, $\mathrm{M}$ and $\overline{\mathrm{q}}$ are not independent of each other and vary in unison along nearly constant $\mathrm{P}_{t}$ $(\mathrm{H})$ lines with changes in the RPM of the TDT main drive motor. The H-line being traversed at any given time is determined by the amount of gas or initial pressure in the tunnel. H-lines between 200 psf and 500 psf were used in the TBW test. The highlighted region of Figure 3 is where all TBW data were acquired and data presented later in the paper will focus on this region.

Finally, it should be pointed out that while the model was scaled for testing in R134a, it is common practice to perform air checkout runs in the TDT prior to filling it with heavy gas. At the tunnel pressures used, tunnel preparation time for air testing was approximately $30 \mathrm{~min}$, while preparation time for heavy gas was approximately $7 \mathrm{hr}$. Returning the tunnel to a condition where personnel could enter the test section took comparable amounts of time. Thus, basic functionality of all systems was verified in air prior to beginning the R134a testing.

\section{Wind-Tunnel Model}

This section of the paper presents a description of the wind-tunnel model, a short discussion of the relevant finite element models along with key modes and frequencies, Ground Vibration Testing (GVT) data acquisition and results, and actuator frequency response.

\section{A. Model Description}

The TBW wind-tunnel model was a fifteen-percent length scale, semispan, sidewall mounted model based on the TBW design described in Reference 3. The model was designed and fabricated by NextGen Aeronautics 


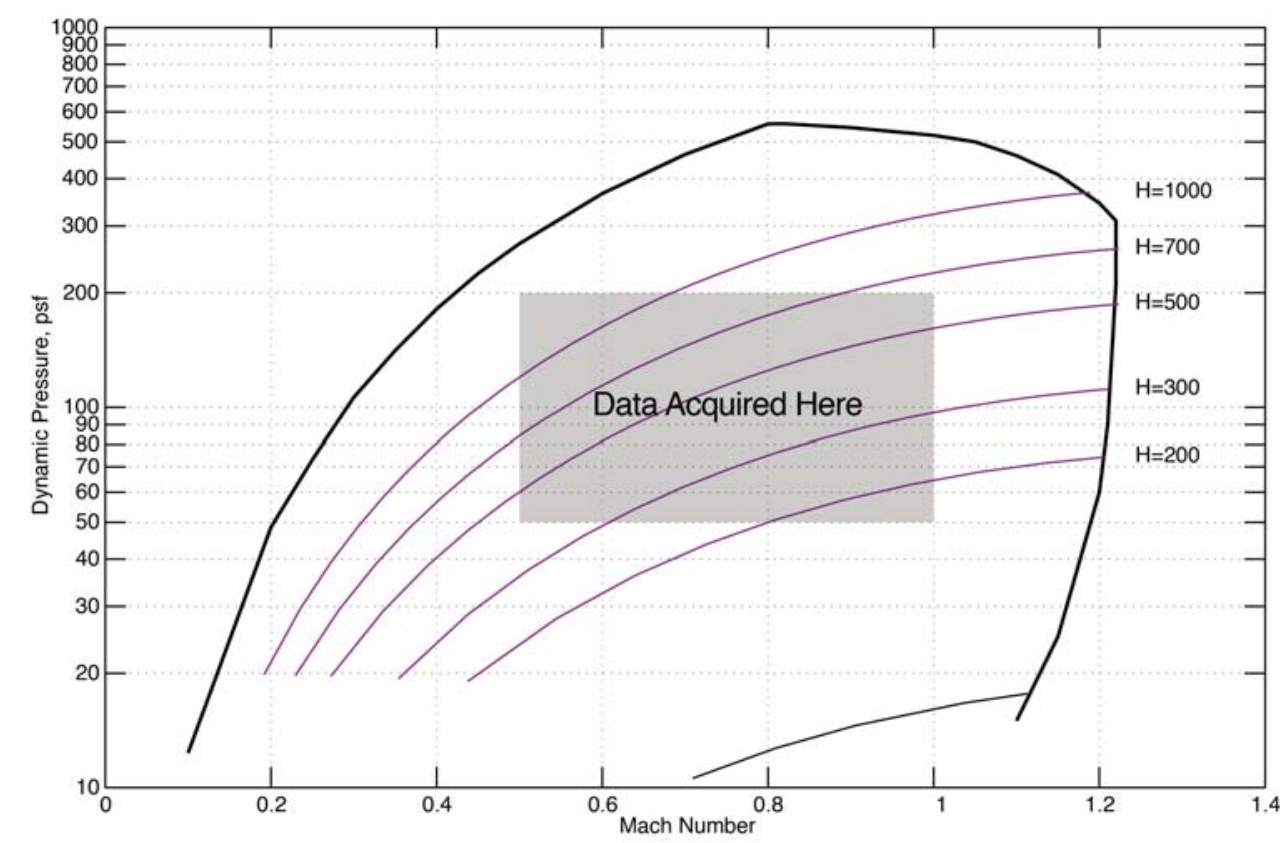

Figure 3. TDT heavy gas operating boundary showing region of TBW testing.

in Torrance, California. The model consisted of several major components including the wing, strut, jury, flow through nacelle, pylon, inboard and outboard ailerons, and fuselage. A photo of the model in the TDT and a labeled image are shown in Figure 4. Strict adherence to the length scaling would have resulted in a fuselage length of $18.7 \mathrm{ft}$; however, since the fuselage was intended only to provide an aerodynamic boundary condition for the wing and associated components, it was shortened to $13.4 \mathrm{ft}$ to reduce model weight and inertia. The model wing span to the vehicle center line was $12.75 \mathrm{ft}$ with an additional 2.25 inches added to the half fuselage (the approximate wind-tunnel boundary layer displacement thickness). The model was supported by a turntable on the east wall of the TDT and the model AOA was varied between $-3^{\circ}$ and $+5^{\circ}$.

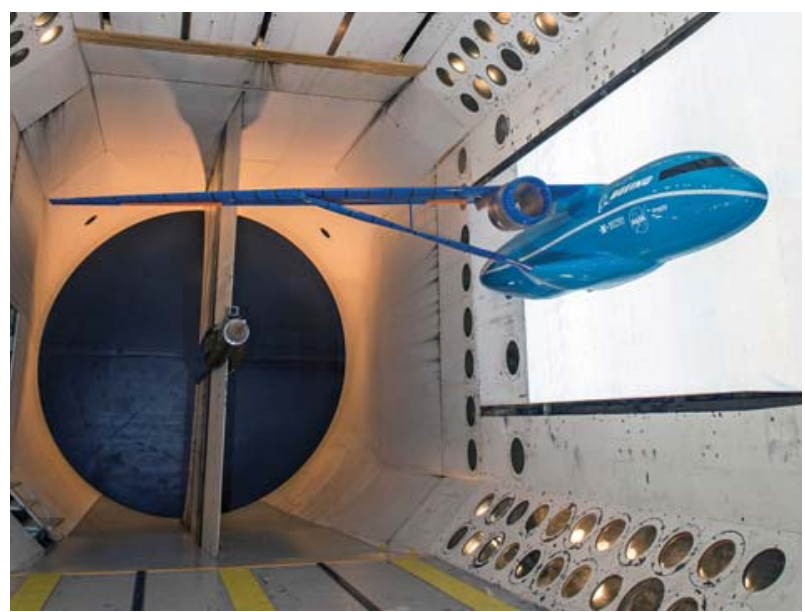

(a) TBW in the TDT

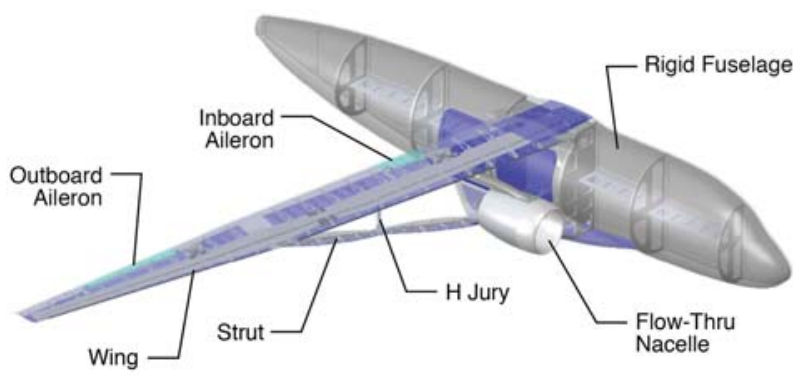

(b) TBW main features

Figure 4. TBW wind-tunnel model in TDT and model features.

The wing, strut, and jury were aeroelastically scaled for testing in heavy gas, which resulted in a heavier, easier to build model with lower elastic mode frequencies minimizing actuator bandwith requirements. The 
components used a spar-pod design where the scaled stiffnesses (EI and GJ distributions) were designed into the flanged aluminum spars, and the aerodynamic shape was provided by discrete fairings, or pods, mounted to the spars. Lead weights were attached to the wing spar in several locations so the wing had the proper scaled mass and moment of inertia. The pods were made of graphite epoxy and attached to the spars using brackets. The nacelle and pylon were not structurally scaled, but had the correct mass and moment of inertia. The fuselage consisted of an aluminum internal structure with fiberglass skin panels.

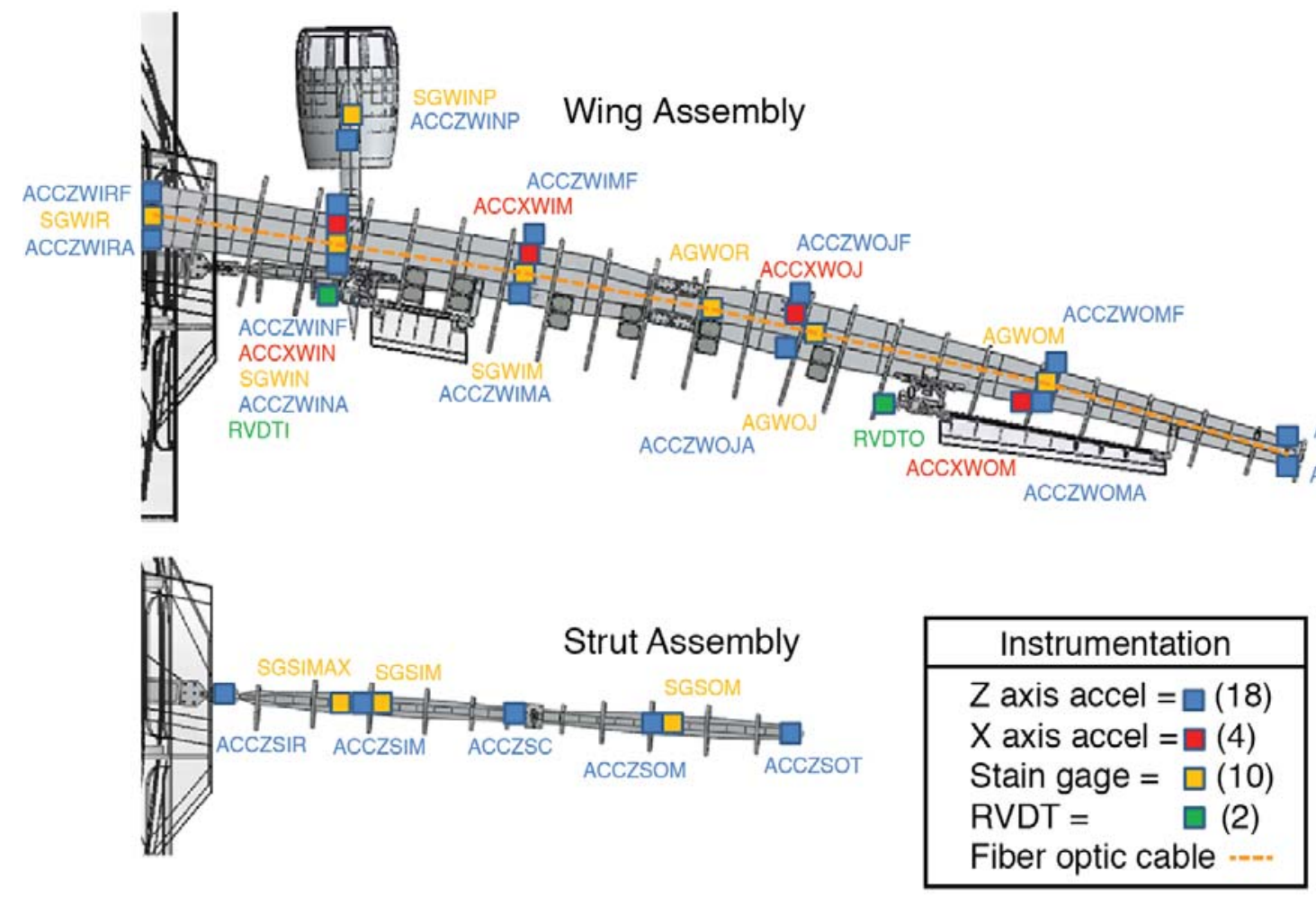

Figure 5. Instrumentation on the TBW model. Not shown is an angle inclinometer used to measure model AOA.

A planform view of the model instrumentation is shown in Figure 5. The primary instruments for measuring aeroelastic response on the TBW model were accelerometers and strain gages. The model had a total of 10 strain gages. All gages were configured to measure strain due to bending except the inboard strut gage (SGSIMAX), which was added later during testing and was configured to measure axial strain. All were full bridge except the half bridge gage on the pylon. There were a total of 22 accelerometers with 18 mounted such that they measured acceleration normal to the spar. For the wing this was vertical, but for the strut the angle was different. The other four accelerometers measured fore-aft acceleration. Finally an angle inclinometer (not shown) was mounted in the fuselage to measure model AOA.

The wing had an inboard and an outboard high bandwidth aileron for use during the ASE portions of the wind-tunnel test. Each aileron was driven by a vane-type hydraulic actuator operating at 1000 psi using a Moog series EO20 servo valve with position measured by a Rotary Variable Differential Transducer (RVDT). This arrangement was very similar to what was used in the AEI Joined Wing SensorCraft test described in Reference 5. The major difference was that the actuator body and vanes were elongated to increase the available torque. Actuator bandwidth is an important consideration for flutter suppression control law design and performance. Here, the goal was to control a $10 \mathrm{~Hz}$ flutter mechanism, so actuator bandwidth had to be maximized and performance monitored through the test. Performance was assessed by sending linear sine sweep excitation signals to the ailerons, acquiring the response data and estimating the frequency response. This was done after model set up to set the actuator gains, and then periodically during the test to monitor performance. Figure 6 shows the frequency response of the actuators both before and after testing. These 
data sets show that the control surface frequency response remained consistent during the TBW wind-tunnel test.
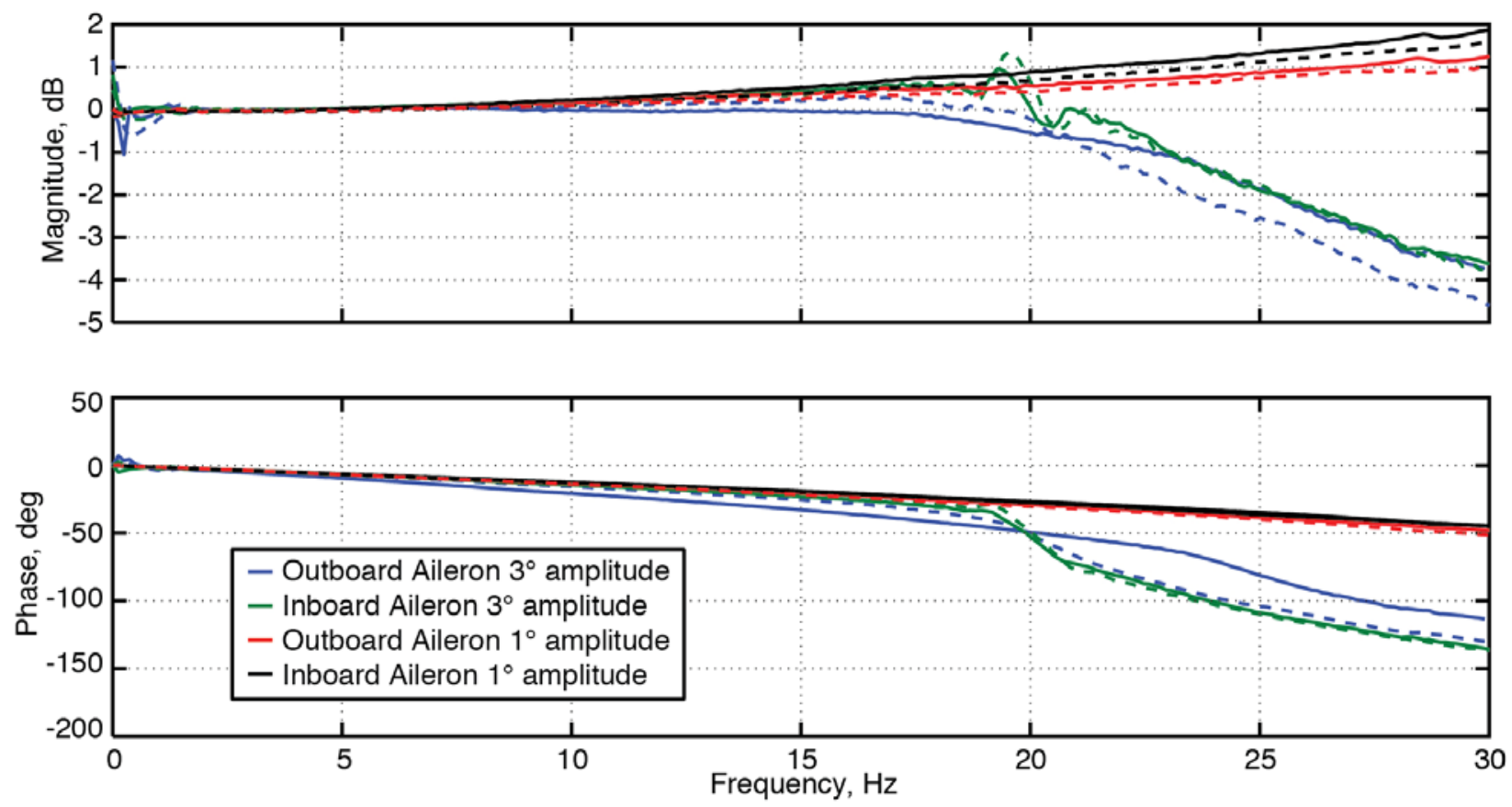

Figure 6. Aileron frequency response function estimates from the start of the test (solid) and the end of the test (dash), linear sine sweep excitations from 0.1 to $30 \mathrm{~Hz},+/-1^{\circ}$ and $+/-3^{\circ}$ amplitude.

\section{B. Ground Vibration Testing and Test Configurations}

Multiple Ground Vibration Tests (GVT) were performed as an important component of the TBW windtunnel test. GVTs were conducted periodically throughout testing in order to validate consistent model behavior and to correlate with The Boeing Company's FEMs. During the pre-test timeframe, GVTs were performed on the model in the Model Prep Area (MPA) followed by an additional GVT once the model was mounted on the wall in the TDT. The shaker set-up for the GVT conducted in the TDT is illustrated in Figure 7. Select flexible modes include a variety of in-plane and out-of-plane wing bending and wing/nacelle torsion. Higher frequency modes also include strut bending and strut torsion. The two primary flutter modes are Mode 3 (second wing bending) and Mode 4 (wing/nacelle torsion). These modes are illustrated in Figure 8. In Figure 8, the NASTRAN modes have been splined to CFD surface mesh for visualization purposes. The contours shown depict vertical deflection. ${ }^{9}$

Testing of the TBW wind-tunnel model took place intermittently over the span of several months (December 2013 - April 2014). Within this testing timeframe, a holiday break temporarily paused testing. Pre-holiday and post-holiday GVT data were acquired. The Boeing Company generated FEMs based on GVT-acquired data. FEM19 was developed based on pre-holiday GVT data and FEM20 was generated based on post-holiday GVT data. A post-holiday GVT revealed a shift in modal frequencies, and it was determined that there was not enough clearance between the strut root joint and the fairing. This determination was tested by looking at GVT results with and without a wedge inserted in a cut-out between the fairing and the inboard strut attachment. A comparison of pre-holiday and post-holiday GVT data and FEM19 and FEM20 frequencies along with modal descriptions is given in Table 1. The differences in post-holiday GVT data compared with pre-holiday GVT data stem from an inadvertent configuration change due to a fouling issue at the root of the strut. Further discussion of the fouling issue and the inadvertent structural configuration change can be found in Reference 3 . 


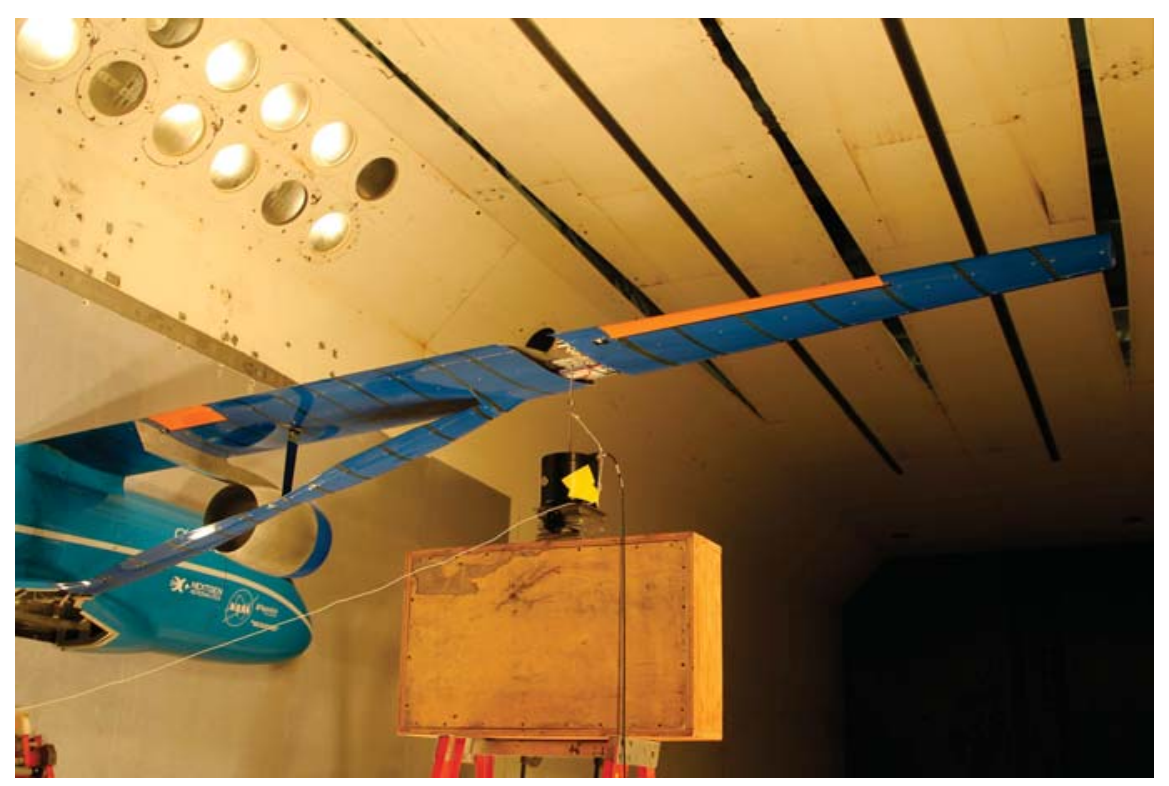

Figure 7. GVT shaker set-up of the TBW wind-tunnel model in the TDT.

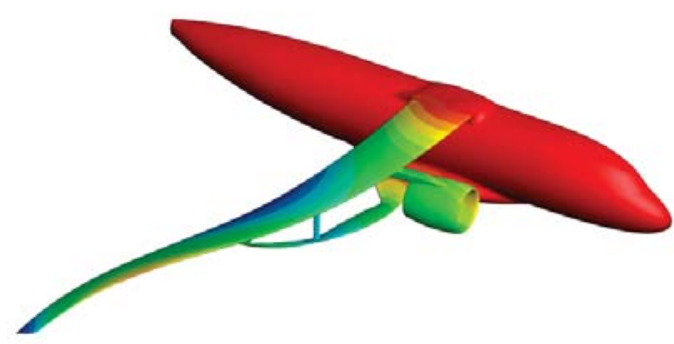

(a) Wing $2^{\text {nd }}$ out-of-plane bending mode

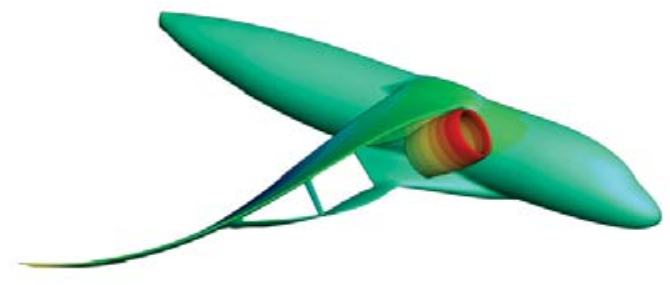

(b) Wing $1^{\text {st }}$ torsion mode

Figure 8. Illustration of TBW flutter mode shapes.

Table 1. Pre-holiday and Post-holiday Modal Data.

\begin{tabular}{c|ccccc}
\hline \hline & \multicolumn{4}{|c}{ Pre-Holiday $(\mathrm{Hz})$} & \multicolumn{2}{c}{ Post-Holiday $(\mathrm{Hz})$} & Description \\
\hline Mode & GVT & FEM19 & GVT & FEM20 & \\
\hline 1 & 5.20 & 5.12 & 5.08 & 5.04 & $1^{\text {st }}$ out-of-plane wing bending \\
3 & 9.08 & 9.17 & 8.43 & 8.44 & $2^{\text {st }}$ out-of-plane wing bending \\
4 & 11.35 & 11.34 & 11.14 & 11.28 & $1^{\text {st }}$ wing/nacelle torsion \\
5 & 19.56 & 18.53 & 18.62 & 18.46 & wing bending \\
7 & 28.44 & 27.44 & 27.57 & 27.13 & wing $/$ nacelle torsion/bending \\
\hline \hline
\end{tabular}




\section{Model Signals, Conditioning, and Control}

This section of the paper will present block diagrams showing increasing levels of detail on how the model signals were routed, conditioned, and controlled. The first of these diagrams shows how signals are routed to the model, the various signal conditioners, the control systems, and the data acquisition system (Figure 9). The second block diagram focuses on the digital control systems (Figure 10). Within these diagrams, signals depicted in orange are operator inputs.

Figure 9 shows a high level schematic of the signal routing arrangement used in the TBW test. As shown, the input and output signals were routed from the model to the TDT control room via the control-roomto-turntable patch panel. Most instrumentation was routed to a Pacific Instruments series 6000 chassis that provided instrument power ( 5 or $10 \mathrm{VDC}$ ), signal amplification, and anti-alias filtering. The anti-aliasing filters were set to $400 \mathrm{~Hz}$ for the RVDTs, as they were routed only to a digital control system (dSPACE1) running at a $1000 \mathrm{~Hz}$ frame rate, and all other signals were filtered at $100 \mathrm{~Hz}$ to be compatible with the other digital control system (dSPACE2) running at a $1000 \mathrm{~Hz}$ frame rate. Servo valve signals were routed to a voltage-to-current converter and back to the model as shown in the figure. Model signals were "teed" and routed to a strip chart for monitoring and to the TDT Data Acquisition System (DAS) for recording. The TDT DAS was set to record data at $500 \mathrm{~Hz}$ with its own anti-aliasing filters set to $200 \mathrm{~Hz}$.

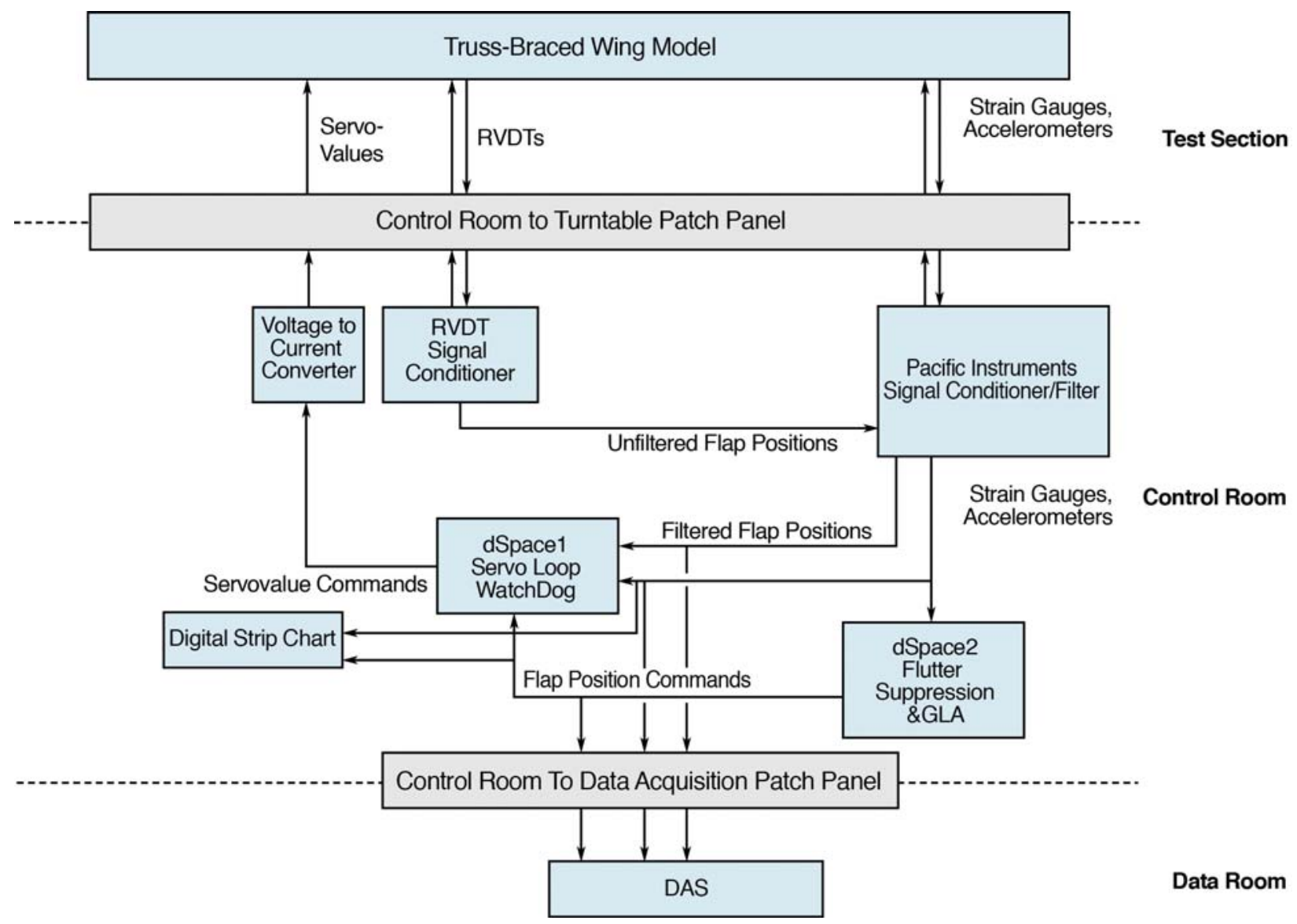

Figure 9. Signal routing used in the TBW wind-tunnel test.

Figure 10 depicts the dSPACE1 and dSPACE2 internal block diagrams and the external signals connecting these systems. All signals external to the dSpace blocks are analog, with dSPACE input signals being converted from volts to engineering units prior to processing and output signals being converted from engineering units back to volts. For signals intended to depict discrete system states, programming logic was used to decode the meaning of the analog signal.

As shown in Figure 10, dSPACE2 incorporated a flight control block and a set of externally generated control surface commands, $\delta_{c m d}$, that could be summed and output as analog control surface command 


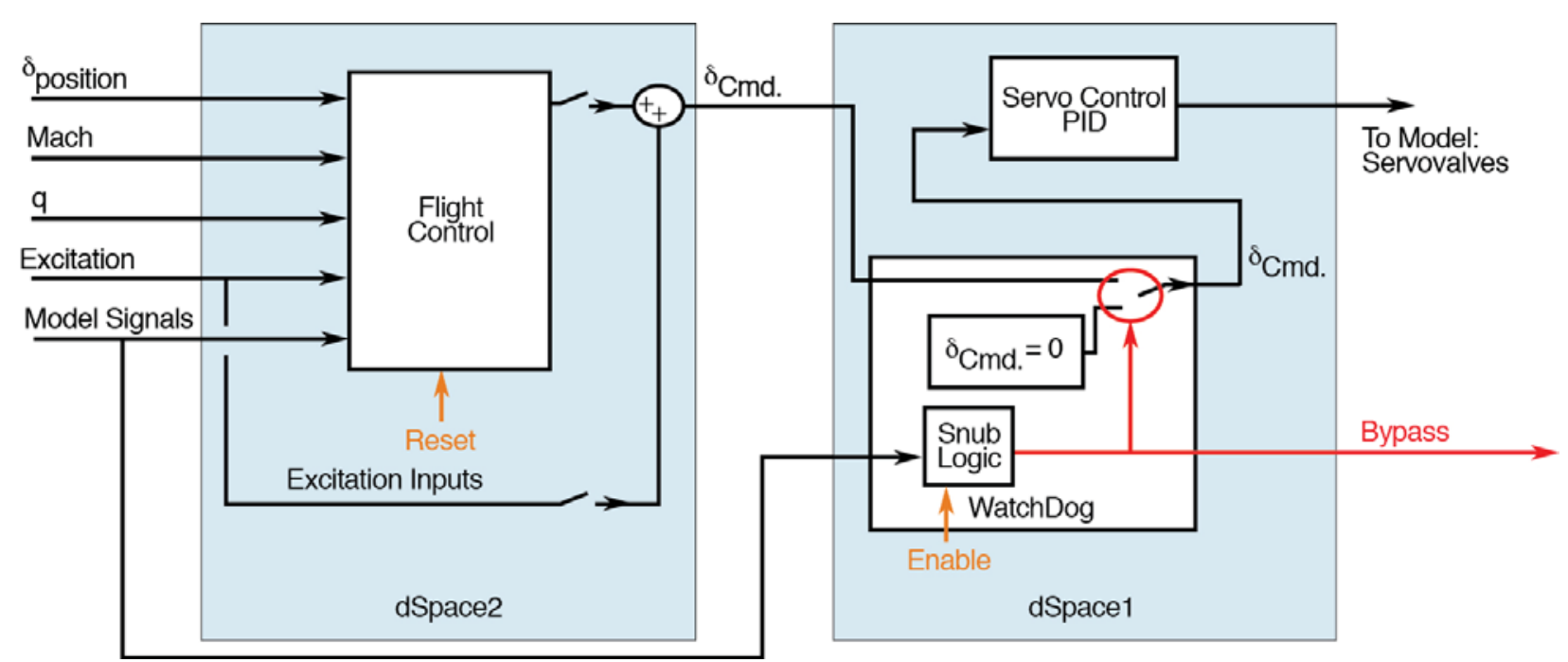

Figure 10. dSpace control block diagram.

signals to dSPACE1. The flutter suppression control laws were implemented within the flight control block. The servo-control loops and the WatchDog system were implemented on dSPACE1. The servo-loops were independent Proportional Integral Derivative (PID) control loops equipped with output saturation blocks to prevent overdriving the actuators. The WatchDog system was an available feature that could be used to monitor the model signals and issue a command when a fault was detected; however, it was not used during the TBW wind-tunnel test. For the TBW test, the emergency controller consisted simply of $0^{\circ}$ control surface commands, and the control block on dSPACE2 had a fault detection algorithm.

\section{A. dSPACE Digital Control Systems}

Each dSPACE Digital Control System (DCS) consists of a rack containing a host computer, a target system, a keyboard, a monitor, Bayonet Neil-Concelman (BNC) patch panels for IO, and an uninterruptible power source. The heart of the DCS is the target system that includes a dSPACE DS1006 control processor board utilizing a $2.6 \mathrm{GHz}$ Advanced Micro Devices (AMD) Opteron processor connected to three dSPACE DS2002 multi-channel A/D boards and one dSPACE DS2103 multi-channel D/A converter board. The A/D boards each have 32 channels utilizing 16-bit quantization with an input range of $\pm 10 \mathrm{~V}$. The $\mathrm{D} / \mathrm{A}$ board contains 32 channels of 14 quantization bits designed for $\pm 10 \mathrm{~V}$ and a settling time of $10 \mu \mathrm{sec}$.

The controller software is developed within the MATLAB Simulink environment, then compiled and downloaded to the target processor via the dSPACE and MATLAB Real-Time Interface. An integral component of the dSPACE tools is the ControlDesk application. ControlDesk provides the user interface to the target processor for the development and implementation of the Graphical User Interface (GUI). The host computer runs the GUI and controls all communications between the processors.

\section{B. RVDT and Servo Valve Signal Conditioning}

As part of the test program described in Reference 5, two custom chassis were assembled at the TDT using commercially available signal conditioning components. These chassis were used to support the TBW test.

The RVDT signal conditioner chassis consisted of 16 LDM-1000 Linear Variable Differential Transformer (LVDT) rail mounted signal conditioner modules and their DC power source manufactured by Schaevitz Sensors. These conditioner modules supplied an AC signal to the RVDTs and then demodulated and amplifed the output. A number of internal dip switches can be used to configure the units. For the TBW test, the RVDTs were driven at $3 \mathrm{~V}$ RMS, and the position output range was set to \pm 5 VDC.

The servo valve signal conditioner chassis consisted of 8 two axis voltage-to-current converters manufactured by Delta Computer Systems. Each of these units transforms $\pm 10 \mathrm{~V}$ signals into current signals capable of driving the Moog hydraulic servo valves. Each unit can drive two servo valves, and full scale current output is selectable with the $\pm 10 \mathrm{~mA}$ setting used for the TBW test. 


\section{TDT Data Acquisition System}

The TDT Open Architecture Data Acquisition System (OADAS) consists of three data acquisition/reduction systems. The Real-Time Systems available to the facility are the Tunnel OADAS (TDTDASE), the Model Preparation Area OADAS (TDTDASD) and the Mobile OADAS (TDTDASM). Together, these computer systems provide the functionality of acquiring, processing, recording and displaying data from the tunnel, the model preparation area, and other locations where a mobile system may be required. These functions are largely identical among all three systems. The Mobile system was used during model preparation and the Tunnel system was using during the wind-tunnel test.

The tunnel data acquisition subsystem is responsible for collecting the data from several different data acquisition units. The analog-to-digital conversions are provided by four NEFF 600 data acquisition units (DAUs). Each DAU can scan up to 64 analog channels at an aggregate rate of almost 100,000 samples per second. In addition to the analog channels, the first DAU also contains digital channels for tunnel parameter and time code data.

\section{ASE Models and Control Law Design}

Two separate control law designs were developed for the closed-loop portion of the TBW wind-tunnel test. Both designs were based on Linear Quadratic Regulator (LQR) design techniques with a Kalman Filter state estimator. Each control law design consisted of a set of several point designs that were scheduled based on $\mathrm{M}$ and $\overline{\mathrm{q}}$. One set of control laws was designed using experimentally derived, system identification (SysID) based state space models (SSM). The other set of control laws was designed using finite element (FEM19) derived SSMs. Both control laws were Multiple-Input and Multiple-Output (MIMO) using all accelerometers except the two at the wing root as inputs and the two aileron commands as outputs. This section of the paper will described both of these designs in the two subsections that follow.

The MATLAB Simulink model used for control law design and evaluation is shown in Figure 11. The actuator block (shown in orange) contains the model of the actuators based on that which was developed for the AEI model. The actuator model attempts to capture the actual displacement as opposed to the RVDTmeasured displacement, which may have some lag. The outputs of the actuator are the inputs to the plant (control surface positions, velocities, and accelerations) and the sensed position of the control surfaces (the RVDT signals). The actuator model is followed by the plant (in state space form), the outputs of which are the accelerometer signals. The primary inputs to the controller (blue) are the accelerometer signals and the RVDT signals. The current tunnel $\mathrm{M}$ and $\overline{\mathrm{q}}$ are input for nearest neighbor gain scheduling. The simulation architecture was designed so that inserting the controller in the dSPACE control system would be as easy as possible. This model was used as shown for the SysID controller development. The FEM19 based designs used this model, but without the sensed position signal as noted in the figure.

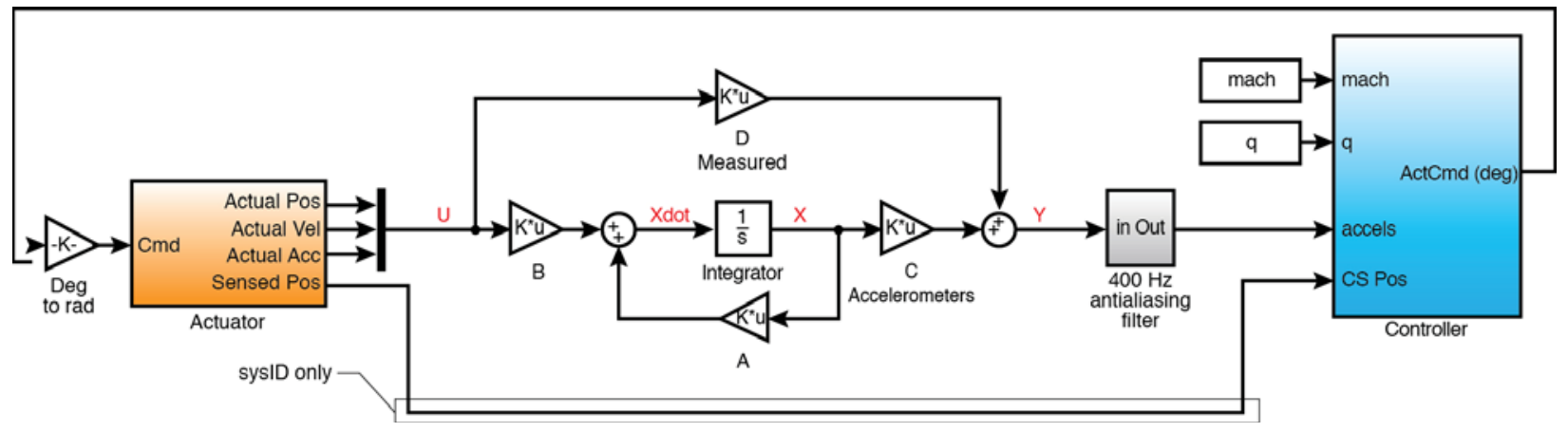

Figure 11. Controller simulation architecture.

\section{A. SysID Based Control Law}

The SysID methods employed to derive the state space models were based on methods developed previously for the AEI program. During the AEI wind-tunnel test, there were several false starts in developing the best approach to using the wind-tunnel data to refine the ASE models. The technique that was ultimately used 
involved the use of an optimizer to tune the simulation model to match the experimental time history data, and details regarding the development of this method can be found in Reference 13.

SysID models were generated for two tunnel conditions, $\mathrm{M}=0.65, \overline{\mathrm{q}}=53.9 \mathrm{psf}$ and $\mathrm{M}=0.70, \overline{\mathrm{q}}=61.6 \mathrm{psf}$. For each model, two sets of actuator sweep time histories (one each for the inboard and outboard ailerons) were used with the simulation model shown in Figure 11. The controller block was not used here. The initial ABCD matrices were based on the FEM19 ASE model described in the next section. An optimizer modified the ABCD matrices until the simulation model output agreed with the experimental model output. The converged matrices were used for control law design.

Figure 12 shows the controller design for the SysID system. The controller is comprised of two major elements. The first, shown in the upper part of the figure, is a nearest neighbor algorithm used to determine which set of Kalman filter (ABCD matrices) and LQR gains to use in the controller. This block has some hysteresis built in to prevent bouncing between sets of gains. The other element is the actual controller, shown in the lower part of the figure. Here, the sensed control surface positions and accelerometer signals are combined and fed through a Kalman filter state space estimator to calculate the estimator outputs X. The resulting outputs from the estimator $(\mathrm{X})$ are multiplied by the LQR gains. A Cmd Filter is then applied to obtain the two control surface commands for output to the actuators.

The estimator coefficients are derived by first designing the full-order estimator gains. The gains are then applied to the SysID model to form a SSM. The SSM is reduced to 10 states (balanced reduction) and discretized at the dSPACE frequency, $1000 \mathrm{~Hz}$, with Tustin pre-warping. The Cmd filter is second-order, 15 $\mathrm{Hz}, 0.65$ damping, Tustin pre-warping, that has been discretized to $1000 \mathrm{~Hz}$.

The LQR gains are derived using a full-order LQR design process primarily weighting the first 5 modes (10 states) with heaviest weighting on the 10 and $20 \mathrm{~Hz}$ modes. The weightings for the LQR gains were tuned to achieve our primary goal of actively damping the $10 \mathrm{~Hz}$ flutter mode while maintaining good stability margins. The final designs were simulated in the time domain and proved to be stable. At the $\mathrm{M}=0.65$ condition, the $10 \mathrm{~Hz}$ mode was attenuated approximately $9 \mathrm{db}$ in the inboard loop and approximately $15 \mathrm{db}$ in the outboard loop, and margins of $9 \mathrm{db}$ gain and $45^{\circ}$ phase were maintained. For the $\mathrm{M}=0.7$ condition, which is closer to the flutter condition and as such was more difficult to control, the margins were slightly lower but still acceptable. Further detail including include Bode and Nichols plots can be found in Reference 3.
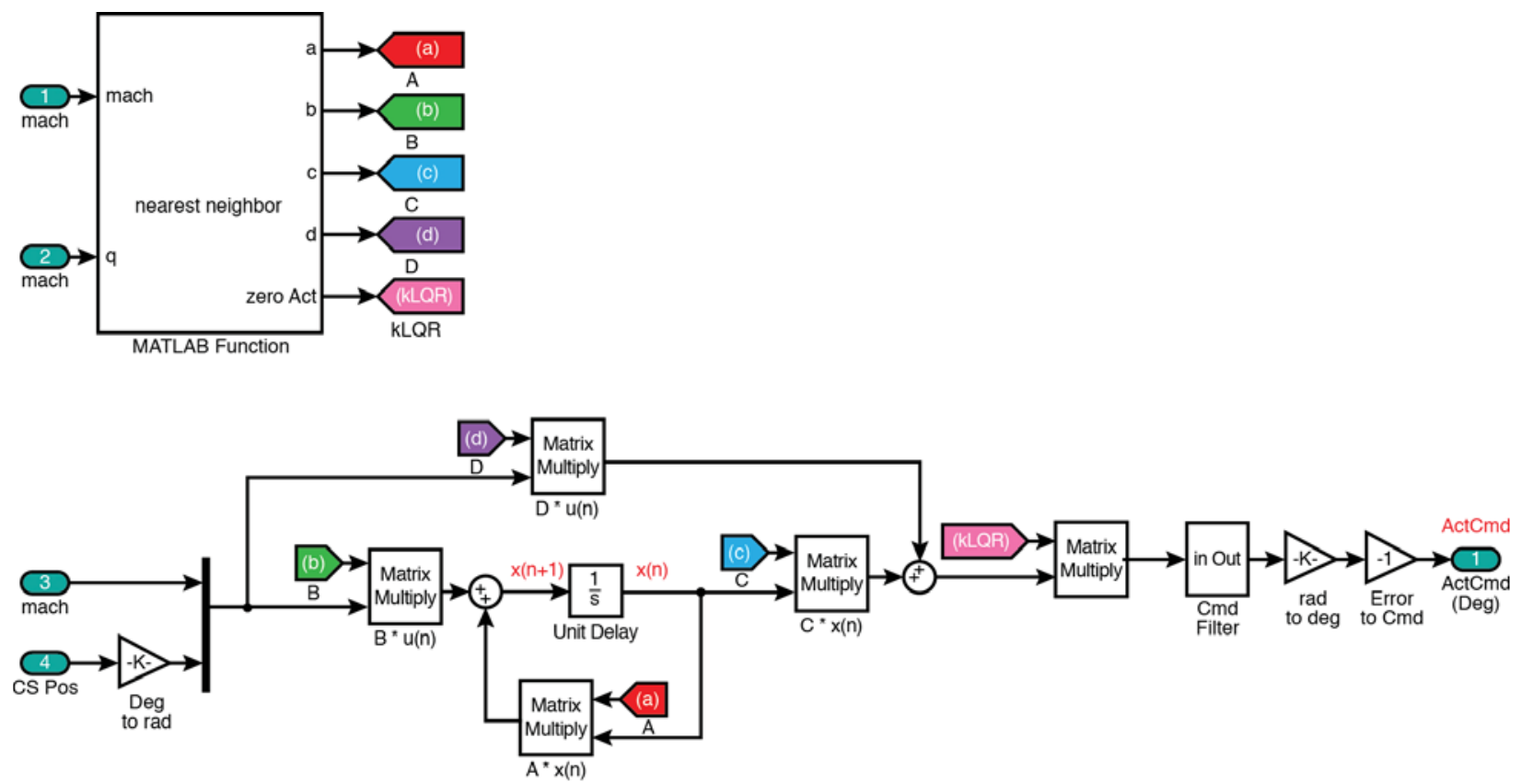

Figure 12. SysID based controller. 


\section{B. FEM Based Control Law}

FEM19 modes and unsteady doublet lattice aerodynamics were used to generate dynamic aeroelastic SSMs, which were used to develop control laws. Unsteady aerodynamics is represented by a P-transform method in the time domain requiring no additional aero states. Models included 40 states for 20 flexible modes up to $100 \mathrm{~Hz}$. Models contain outputs at all accelerometer locations consisting of displacement, rate, and acceleration in all three coordinate directions and rotational displacement and rate about all three coordinate directions. Models contain inputs consisting of the inboard and outboard wing trailing edge control surface deflection, rate, and acceleration to accommodate coupling actuator dynamics. Final FEM19 based models include 13 dynamic pressures at $\mathrm{M}=0.75$ and two dynamic pressures at $\mathrm{M}=0.6,0.65$, and $0.70 .{ }^{3}$

Figure 13 shows the FEM19 based controller. While the FEM19 controller has essentially the same architecture as the SysID based controller, there is a lack of LQR gains in the diagram. The gains still exist; however, they are imbedded in the estimator realization prior to calculating the estimator coefficients. Also, a $15^{\circ}$ limit is imposed on the actuator commands, and the hysteresis in the nearest neighbor algorithm has been modified such that the states and actuator commands could be zeroed out briefly when transitioning between design points. The Cmd filter frequency was increased to $40 \mathrm{~Hz}$ (second-order, $40 \mathrm{~Hz}, 0.65 \mathrm{damping}$, Tustin pre-warping, that has been discretized to $1000 \mathrm{~Hz}$ ).

The process for deriving the LQR and estimator gains is fundamentally different from the SysID version. In order to increase stability margins, the FEM19 state space model was augmented with a pair of secondorder filters (40 Hz, 0.98 damping) to model the two actuator states and allow the LQR process to put gains on them. In order to keep the LQR gains on the actuator states in check, the system was augmented with a pair of third-order filters, effectively penalizing very high gains, to prevent driving the actuator bandwidth too high because of its inherent nonlinearities. The third-order filter is formed by multiplying a second-order filter by a first-order filter $(101 \mathrm{~Hz})$. The second-order filter has a second-order polynomial $(10 \mathrm{~Hz}, 0.6$ damping) in the numerator and a second-order polynomial in the denominator (100 Hz, 0.7 damping). The LQR gains are derived using a full-order LQR design process on the augmented model primarily weighting the first 5 modes with heaviest weighting on the $10 \mathrm{~Hz}$ flutter mode. It was found that heavily weighting the third state of the third-order filter kept the actuator gains within the desired range.

The estimator gains are calculated and the LQR gains are embedded into the estimator to form a new SSM. This model is reduced (balanced reduction) to 20 states (10 modes) and discretized at $1000 \mathrm{~Hz}$. The weightings for the LQR gains were tuned to actively damp the $10 \mathrm{~Hz}$ flutter mode while maintaining good stability margins. For the $\mathrm{M}=0.7$ condition, the $10 \mathrm{~Hz}$ mode was attenuated about $7 \mathrm{db}$ in the inboard loop and about $6 \mathrm{db}$ in the outboard loop, with margins of $9 \mathrm{db}$ and $45^{\circ}$. Analysis of other conditions indicated that the margins were maintained for all but the two most unstable cases $(\mathrm{M}=0.75, \overline{\mathrm{q}}=137.7 \mathrm{psf}$ and $\mathrm{M}=0.75$, $\overline{\mathrm{q}}=160.6 \mathrm{psf}$ ), and margins of $6 \mathrm{db}$ and $30^{\circ}$ were maintained in those cases. Further detail including Bode and Nichols plots can be found in Reference 3.

For the TBW wind-tunnel test, the LQR design process worked very well, allowing the $10 \mathrm{~Hz}$ flutter mode to be targeted directly in the gain design process. A comparison of the SysID model modal frequencies and damping, depicted in Figure 14, shows that for the M=0.65 model, the FEM19 SSM counterpart is in fair agreement, but that the FEM19 model tends to be more attenuated in the 5 to $10 \mathrm{~Hz}$ range. The FEM19 SSMs proved to be sufficient for designing a robust control system, potentially reducing the need for open-loop testing in the future.

\section{Wind-Tunnel Testing}

Testing of the TBW wind-tunnel model took place intermittently over the span of several months (December 2013 - April 2014). As previously mentioned in the model description section of the paper, within this testing timeframe, a holiday break temporarily paused the testing and upon resuming a fouling problem was identified and fixed. This model change effectively resulted in a configuration change and the two configurations are herein referred to as pre-holiday or post-holiday. With the exception of several air checkout runs, all TBW data were acquired in heavy gas, and the air data will not be discussed in this paper.

This section of the paper will describe the testing procedures and experimental results. The open-loop testing procedures and results will be discussed first. This will include flutter, system identification, and

dwells. Next, the closed-loop testing and results will be discussed. This will include flutter suppression, gust response, and closed-loop system identification. 

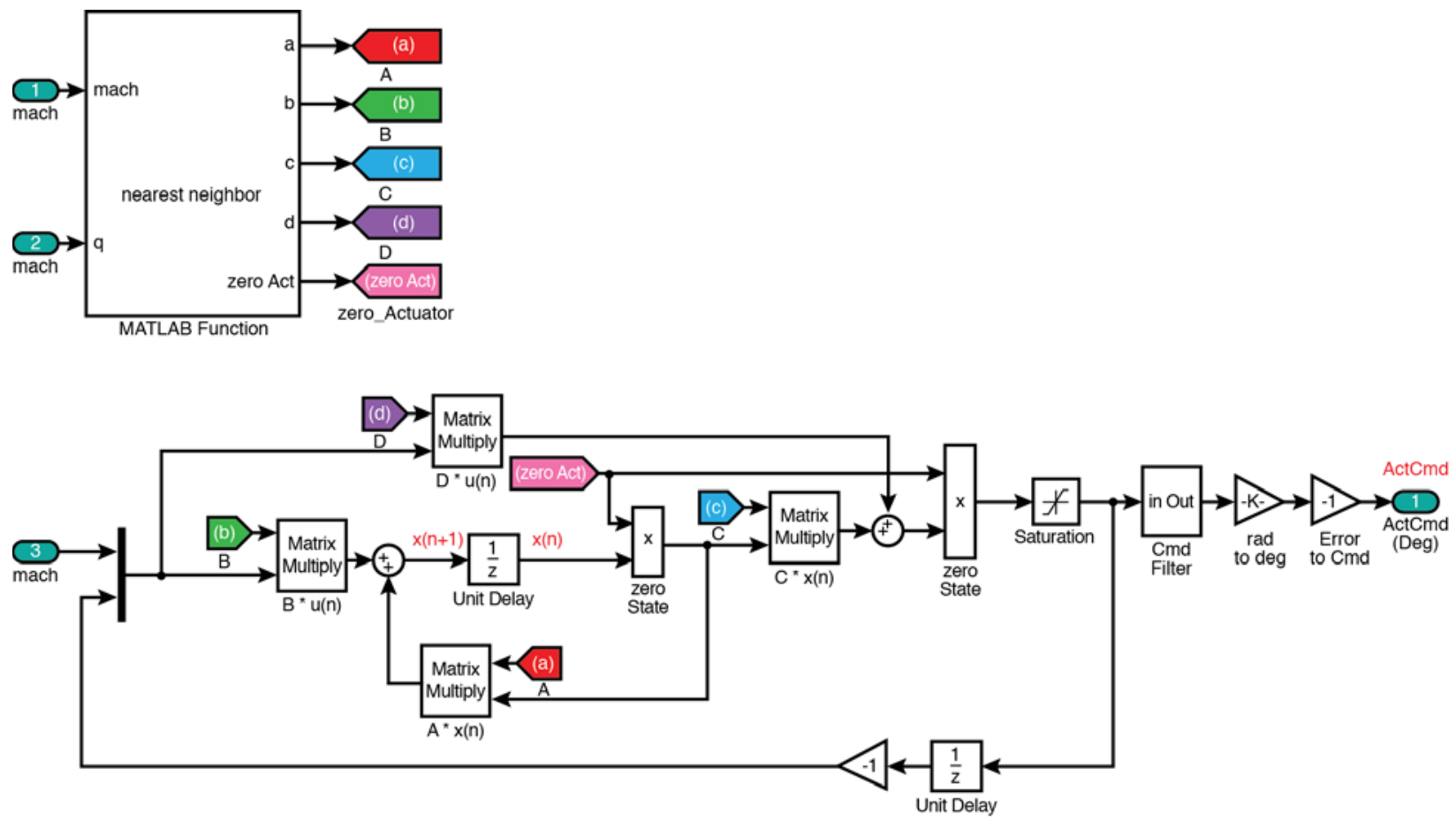

Figure 13. FEM19 based controller.
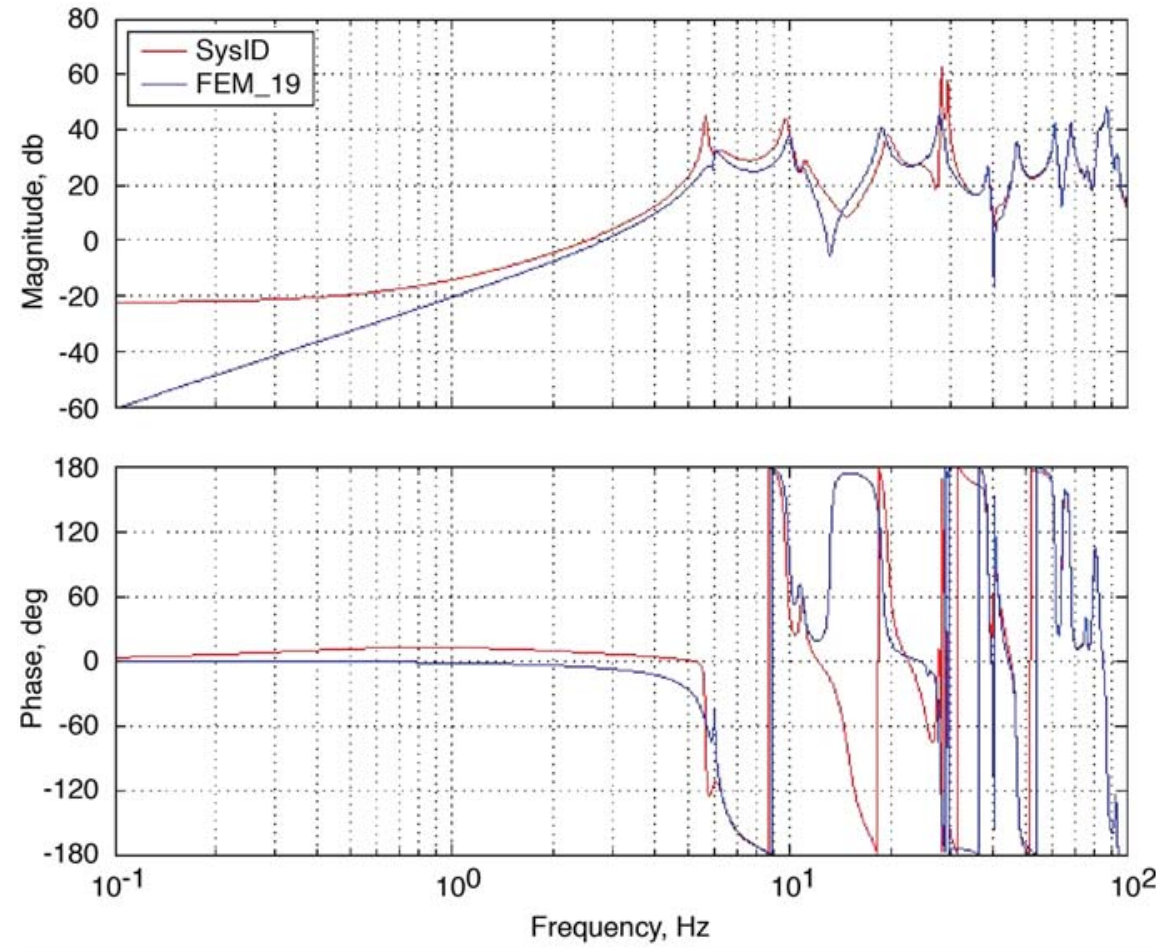

Figure 14. Comparison of Boeing SysID and FEM19 models. Inboard control surface to ACCZWOMF (M=0.65 $\overline{\mathbf{q}}=53.9$ psf). 


\section{A. Open-Loop Flutter Testing}

For open-loop flutter testing, the goal was to establish the $M$ and $\bar{q}$ of flutter onset. Typical flutter testing in the TDT relies on naturally occurring tunnel turbulence to perturb the model. $M$ and $\bar{q}$ are gradually increased along an H-line until a flutter point is obtained. Time history data are generally acquired along each H-line up to and including the flutter point. The determination of flutter is made visually by the test engineers, from strip chart observations, or from other stability assessments. Once a flutter point is obtained, $\mathrm{M}$ and $\overline{\mathrm{q}}$ are reduced to stabilize the model by gradually reducing main drive RPM or by opening the tunnel bypass valves if warranted by the severity of the instability. Repeating this process over multiple H-lines allows the test engineers to define the flutter/LCO onset boundary.

Figure 15a shows stable and unstable data points for the pre-holiday model at an AOA of $-3^{\circ}$ along multiple H-lines. The process was repeated for three other AOAs: $-1^{\circ},+1^{\circ}$, and $+3^{\circ}$. In all cases, the flutter points identify tunnel conditions where the TDT bypass valves were used. Figure 15b shows the pre-holiday flutter points for the four AOAs. The flutter onset boundary is clearly a function of AOA. This nonlinear effect can be captured with NASTRAN using the procedure described in Reference 8. There, the linear flutter analysis uses the updated mass and stiffness matrices from a nonlinear static aeroeleastic solution at the tunnel conditions. The mode shapes and frequencies are a function of wing loading for the TBW.

The post-holiday flutter data for the same four AOAs will be discussed next. For each angle, all open-loop stable points are plotted on the TDT heavy gas operating envelope. Here, tunnel conditions where stable points were not obtained represent unstable regions, and approximate flutter onset boundaries are drawn on the plots. For several AOAs, flutter suppression control laws were used to pass through the unstable region that the H-lines intersect and get to stable tunnel conditions, at higher Mach numbers, in order to define the so-called back side of the onset boundary.

Figure 16 presents the post-holiday flutter data for $-3^{\circ}$ AOA. Here, the upper plot showing all stable points acquired indicates a small unstable region approximately between $\mathrm{M}=0.73$ and $\mathrm{M}=0.78$ and dynamic pressures between 70 and $90 \mathrm{psf}$. The stable points above the unstable region indicate that the flutter mechanism is likely a hump mode as it re-stabilizes at higher dynamic pressures. The lower part of this unstable region appears to be a traditional transonic dip at $\mathrm{M}=0.75$ and $\overline{\mathrm{q}}=71 \mathrm{psf}$. The lower plot shows the same flutter onset boundary and identifies points where the bypass valves were used or limit cycle oscillation was observed. From a qualitative point of view, this AOA proved to be the most inconsistent of all four angles. This was especially true above the triangular flutter region where a mix of stable points, LCO points, and unstable points were acquired. These data were acquired on different days, runs, and H-lines. If the flutter mechanism is indeed a hump mode, small variations in AOA, temperature, and other factors could account for the inconsistent results in this region.

Figure 17 presents the post-holiday flutter data for $-1^{\circ}$ AOA. Here, the upper plot showing all stable points acquired implies a flutter onset boundary with a more traditional transonic dip at a $\mathrm{M}=0.77$ and $\overline{\mathrm{q}}=76$ psf. The lower plot shows the same flutter onset boundary plus open-loop unstable points, LCO points, and points where a functioning control law was disengaged and the model was unstable. There were some inconsistencies with a few unstable points being acquired that are to the left of the onset boundary and some LCO points acquired that are below the onset boundary.

Figure 18 presents the post-holiday flutter data for $+1^{\circ} \mathrm{AOA}$. As with the AOA $-1^{\circ}$ data, a traditional transonic dip in the flutter onset boundary is revealed by examining the open-loop data points in the upper plot. The low point of the dip occurs at a $\mathrm{M}=0.79$ and $\overline{\mathrm{q}}=77 \mathrm{psf}$. The lower plot shows the same flutter onset boundary plus open-loop unstable points, LCO points, and points where a functioning control law was disengaged and the model was unstable. Here, the open-loop unstable/closed-loop stable points indicate that the model remains unstable from the transonic dip up to a dynamic pressure of at least $113 \mathrm{psf}$. The green symbols indicate torsion mode LCO of approximately $11 \mathrm{~Hz}$ at Mach numbers near 0.76.

Figure 19 presents the post-holiday flutter data for $+3^{\circ}$ AOA. Here, the plot of open-loop stable points reveals a probable transonic dip at approximately $\mathrm{M}=0.795$ and $\overline{\mathrm{q}}=91 \mathrm{psf}$. The lower plot shows the same flutter onset boundary plus LCO points. The higher flutter $\overline{\mathrm{q}} \mathrm{s}$ associated with this AOA resulted in an increase in testing risk, and as a result, less data were acquired, and the flutter onset boundary was not defined. 


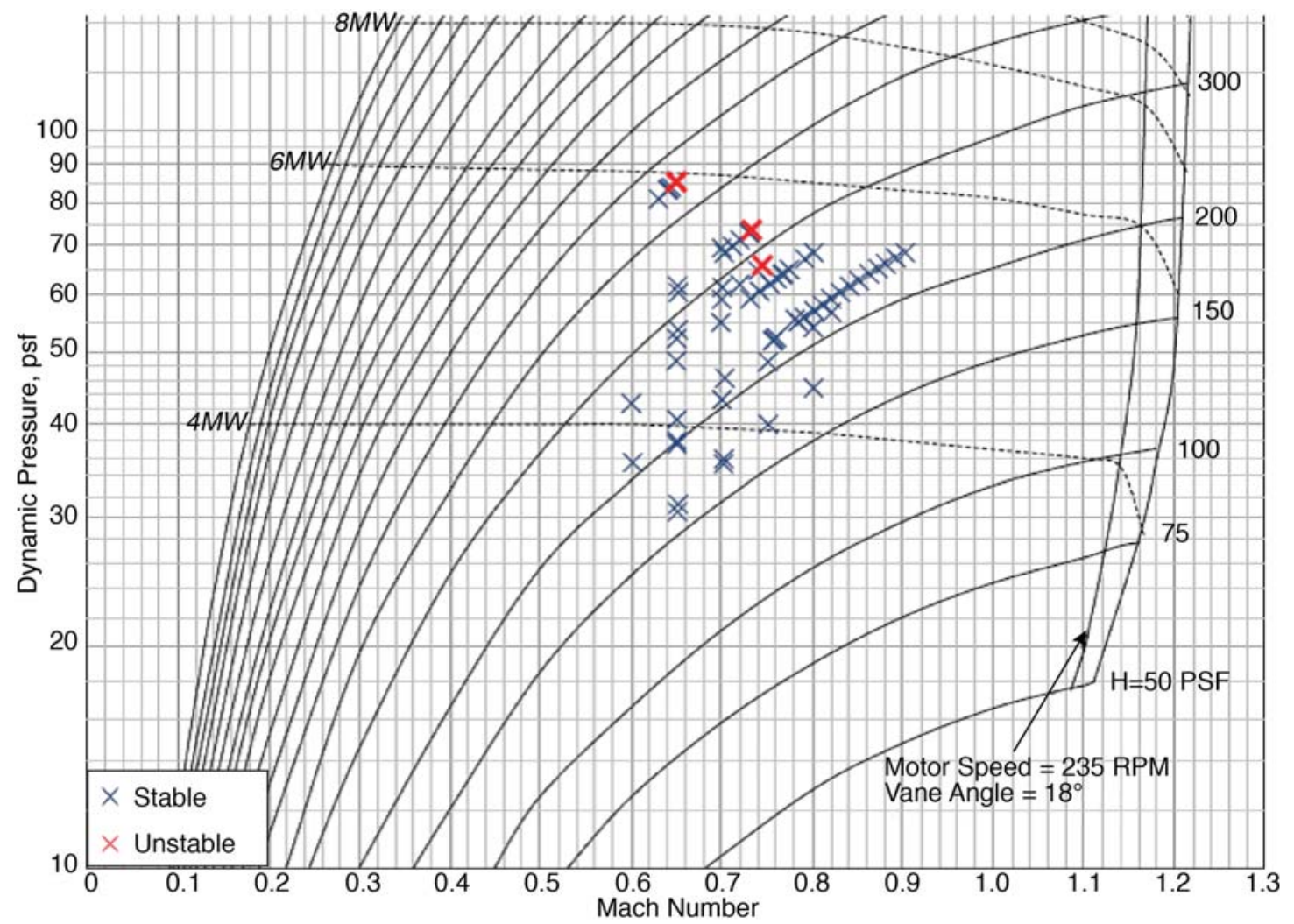

(a) Pre-holiday flutter boundary, AOA $-3^{\circ}$

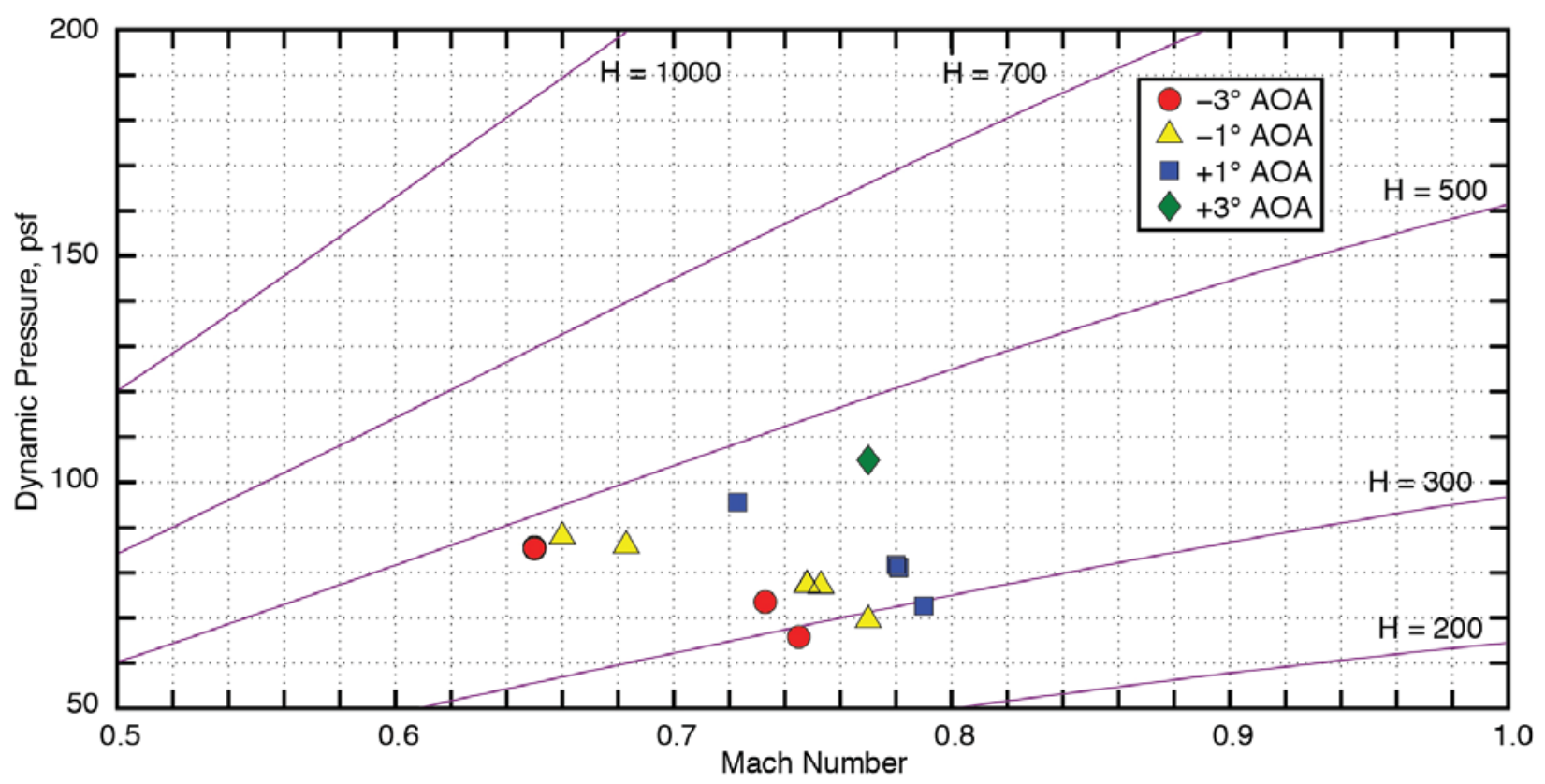

(b) Pre-holiday flutter boundary for all AOAs

Figure 15. Pre-holiday experimental flutter results. 


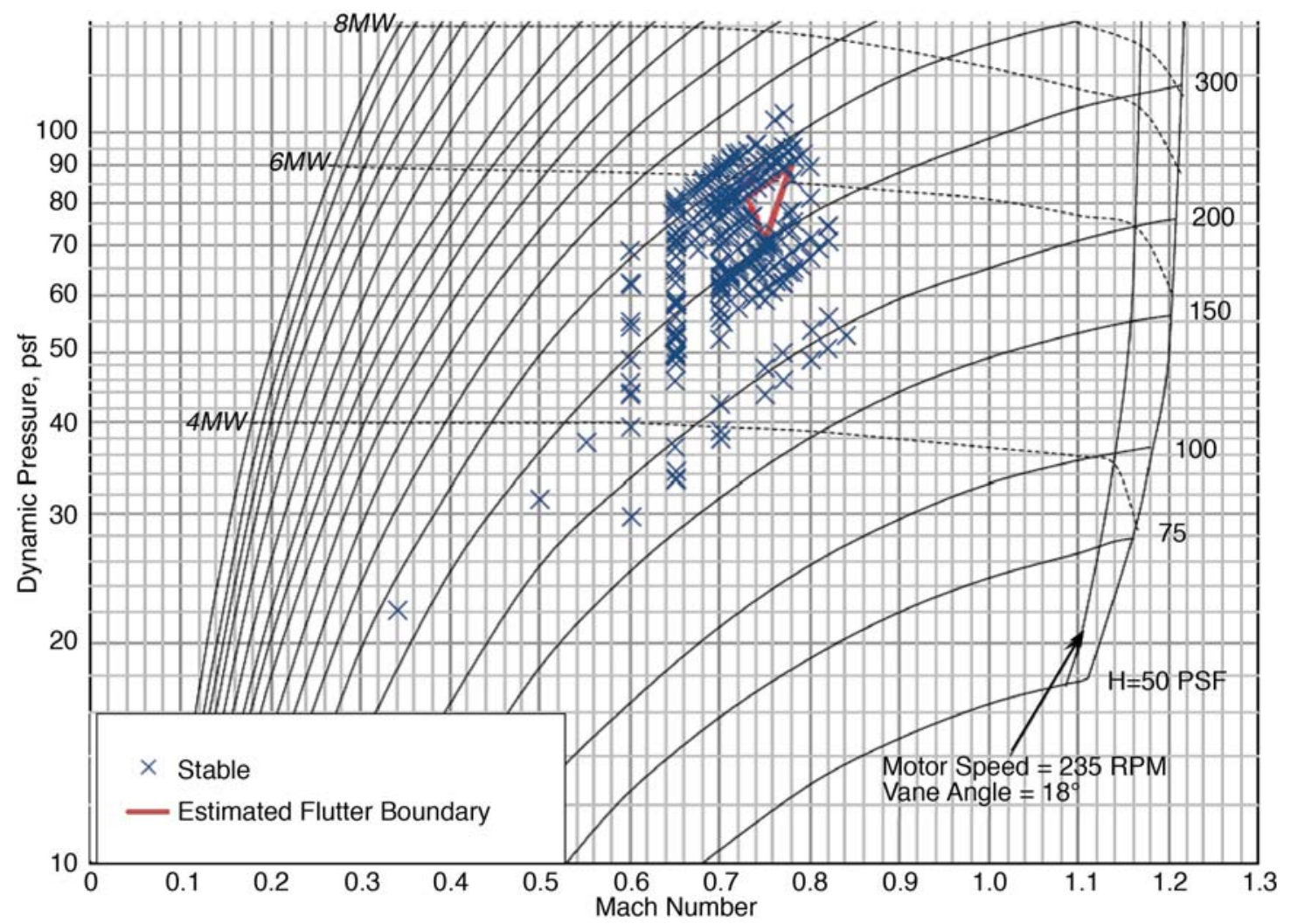

(a) TDT heavy gas boundary with stable points and flutter onset boundary

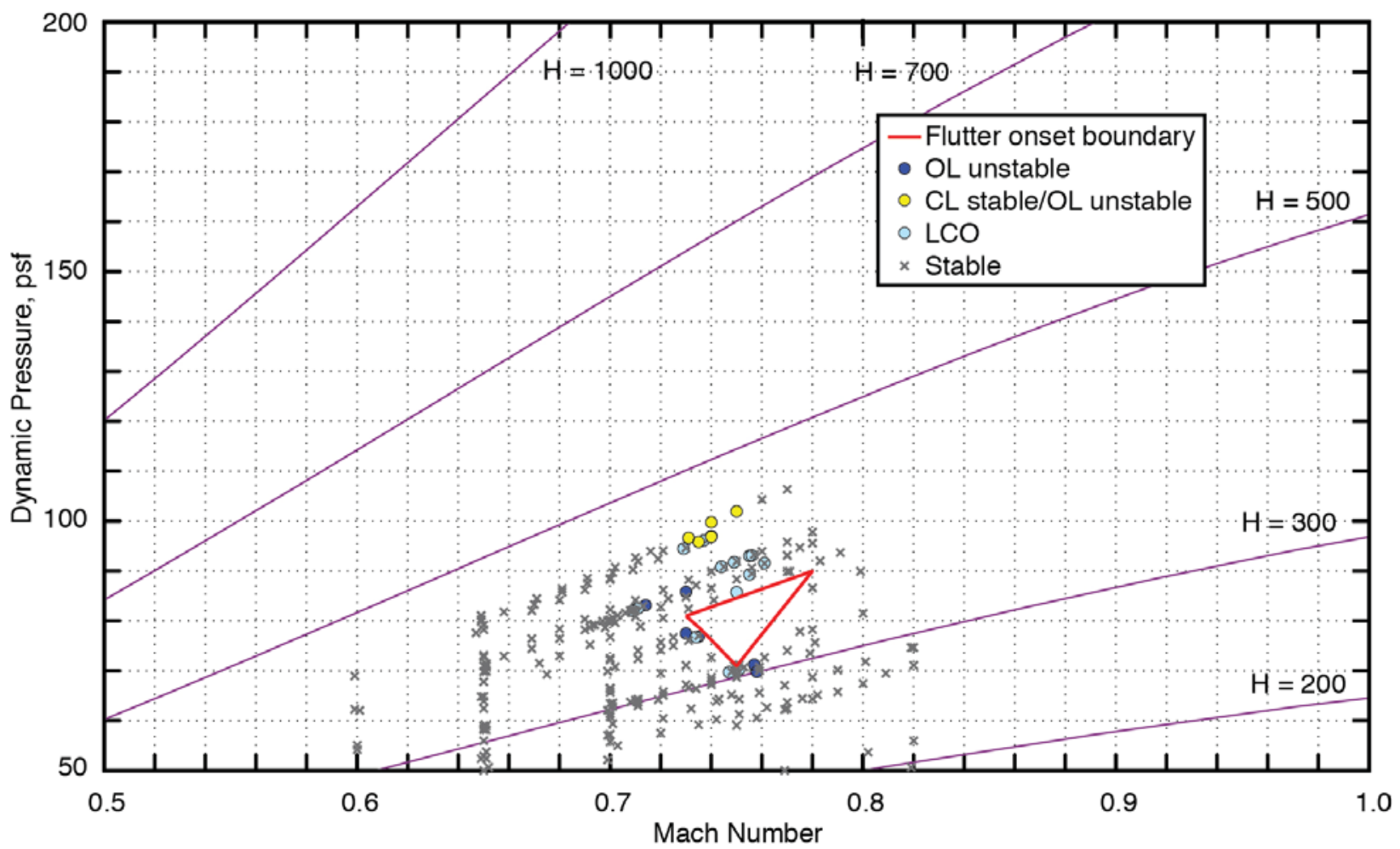

(b) Close-up of onset boundary with LCO and flutter points

Figure 16. Post-holiday flutter onset for AOA $-3^{\circ}$. 


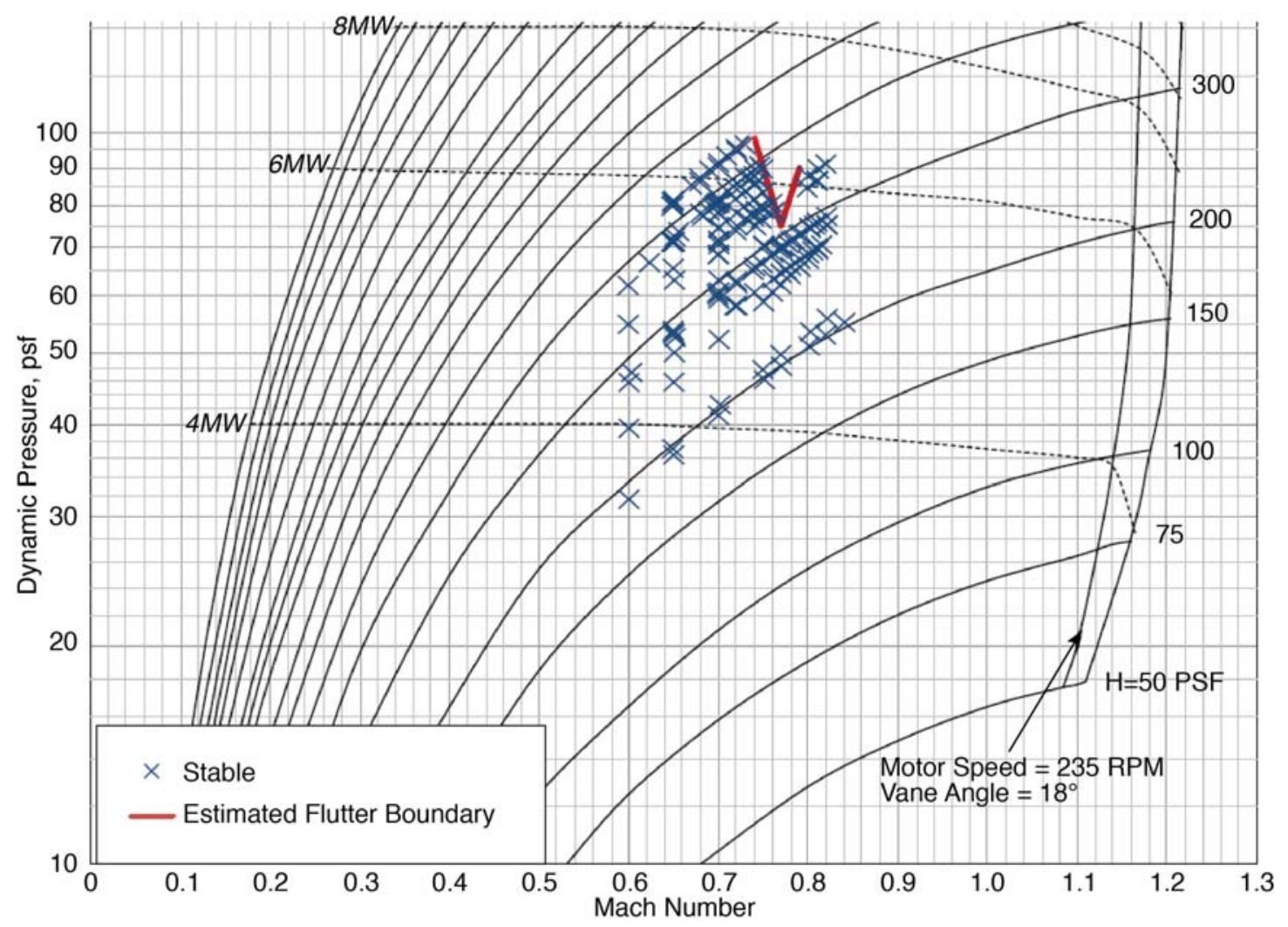

(a) TDT heavy gas boundary with stable points and flutter onset boundary

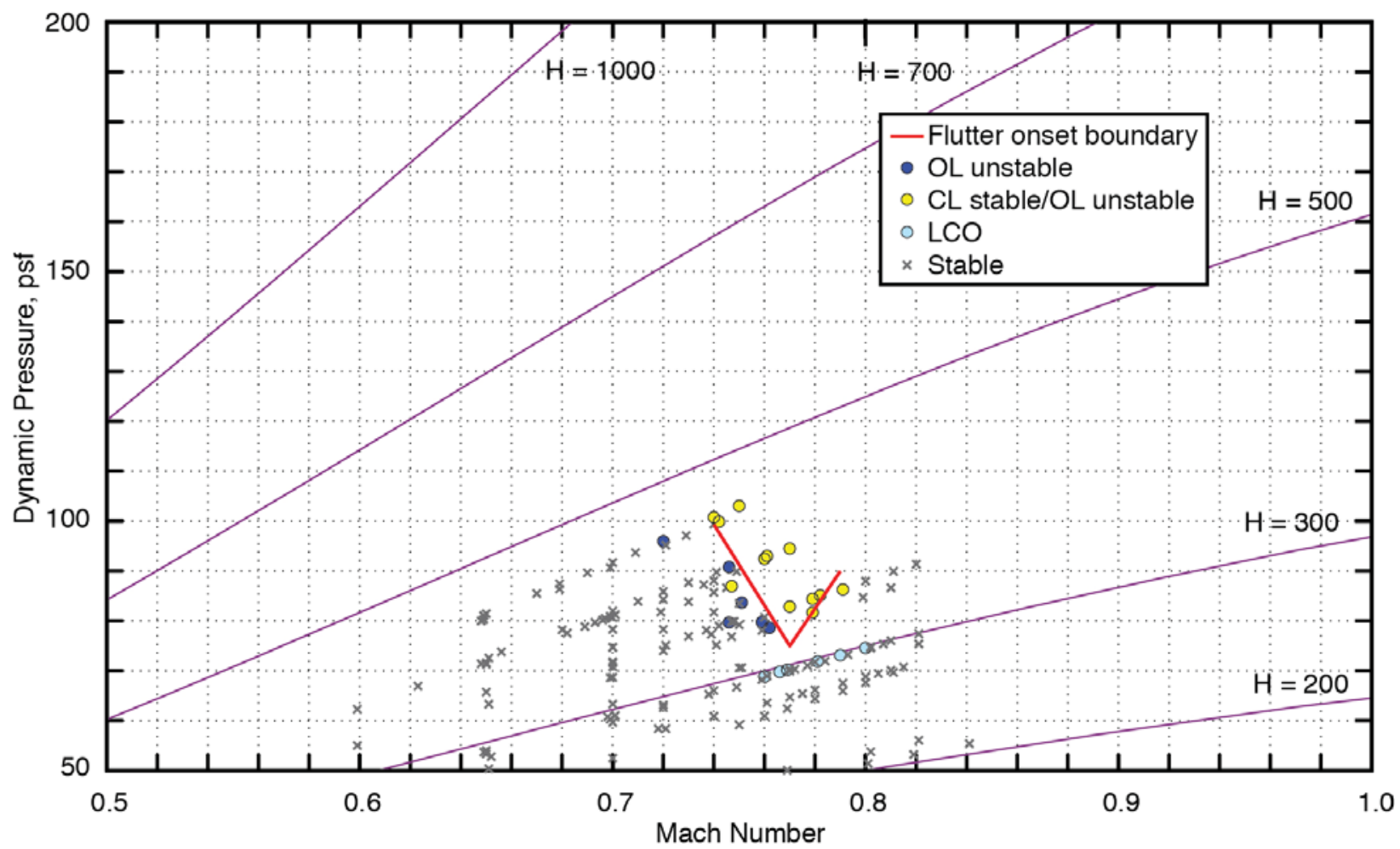

(b) Close-up of onset boundary with LCO and flutter points

Figure 17. Post-holiday flutter onset for AOA $-1^{\circ}$. 


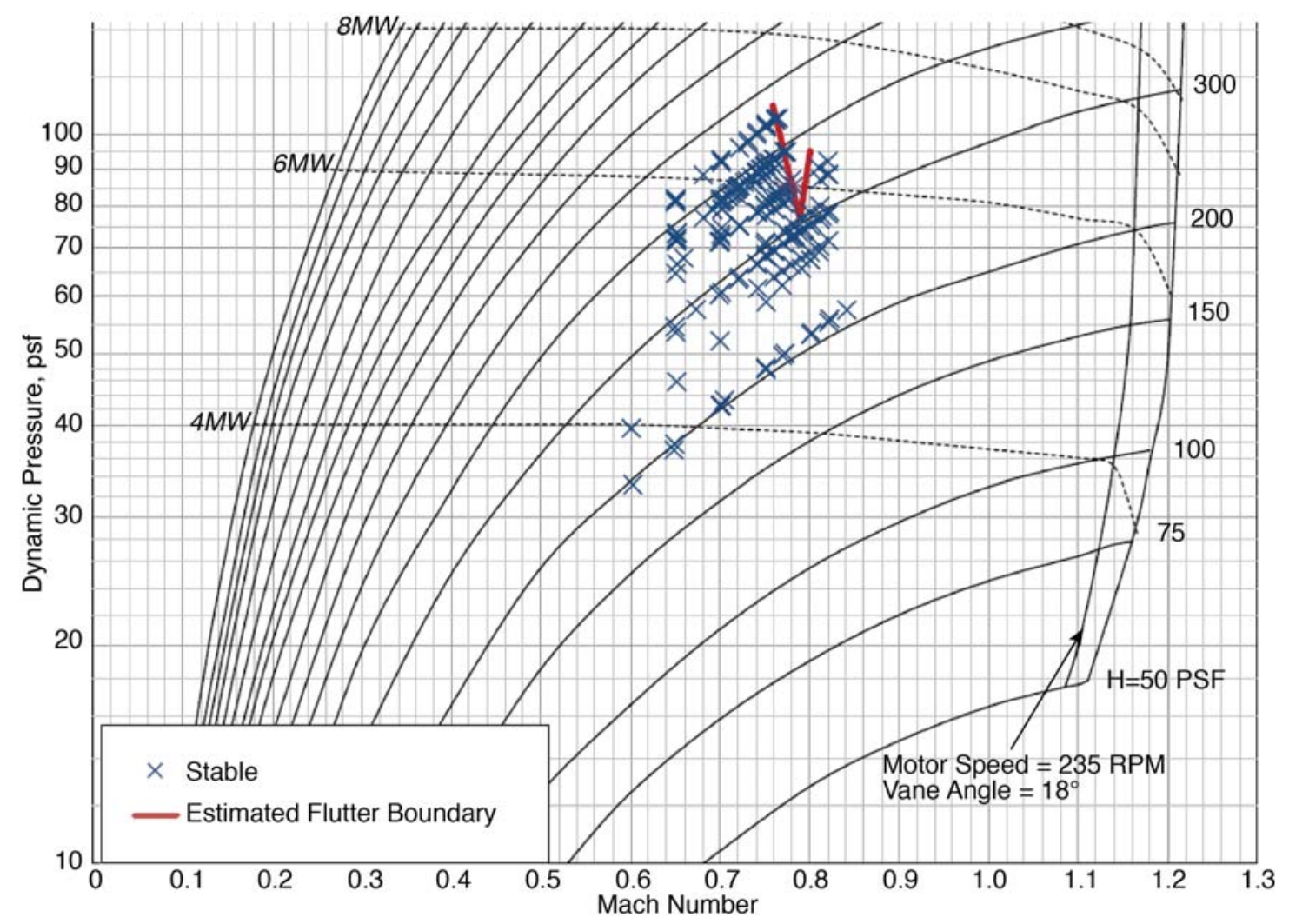

(a) TDT heavy gas boundary with stable points and flutter onset boundary

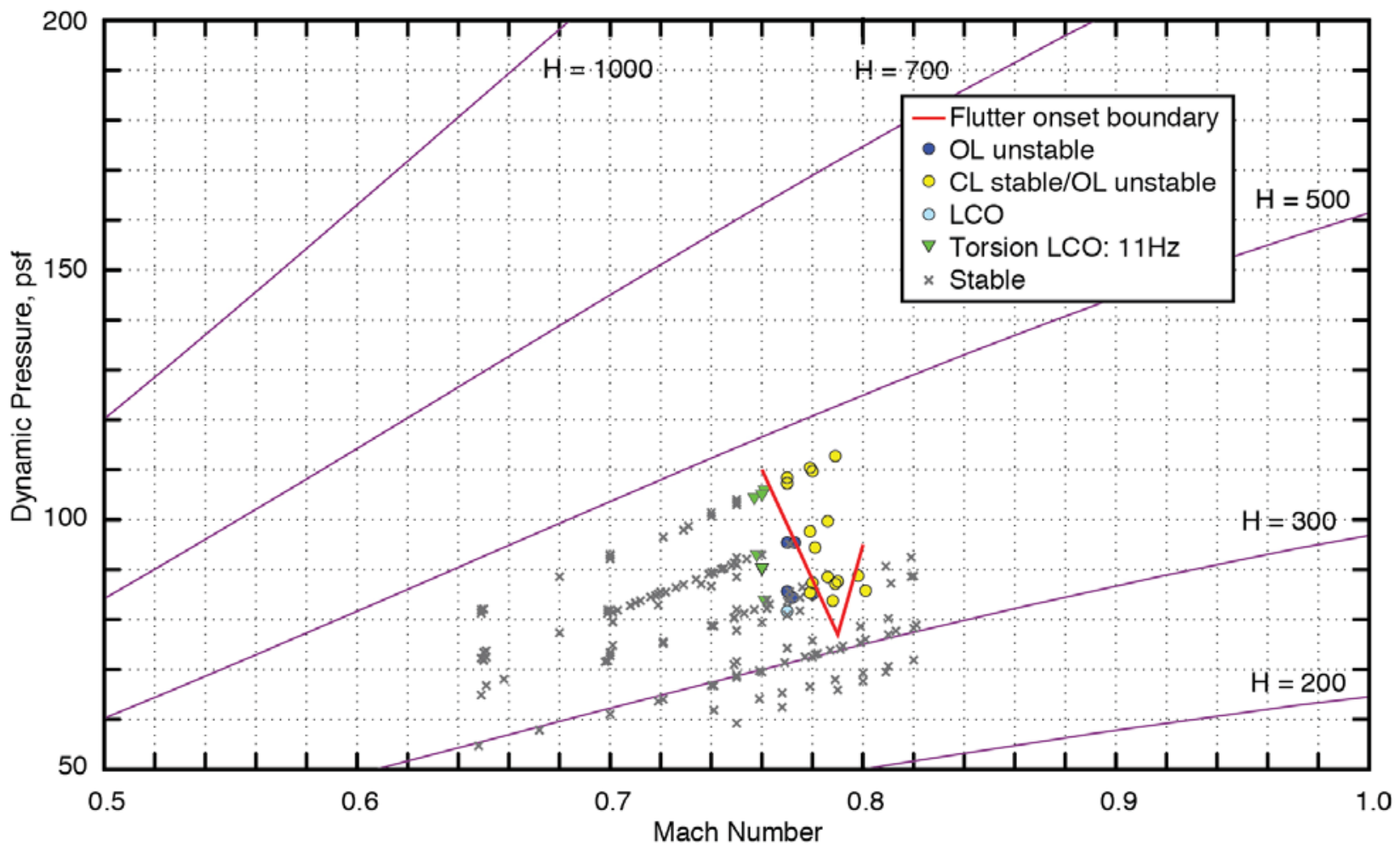

(b) Close-up of onset boundary with LCO and flutter points

Figure 18. Post-holiday flutter onset for $\mathrm{AOA}+1^{\circ}$ 


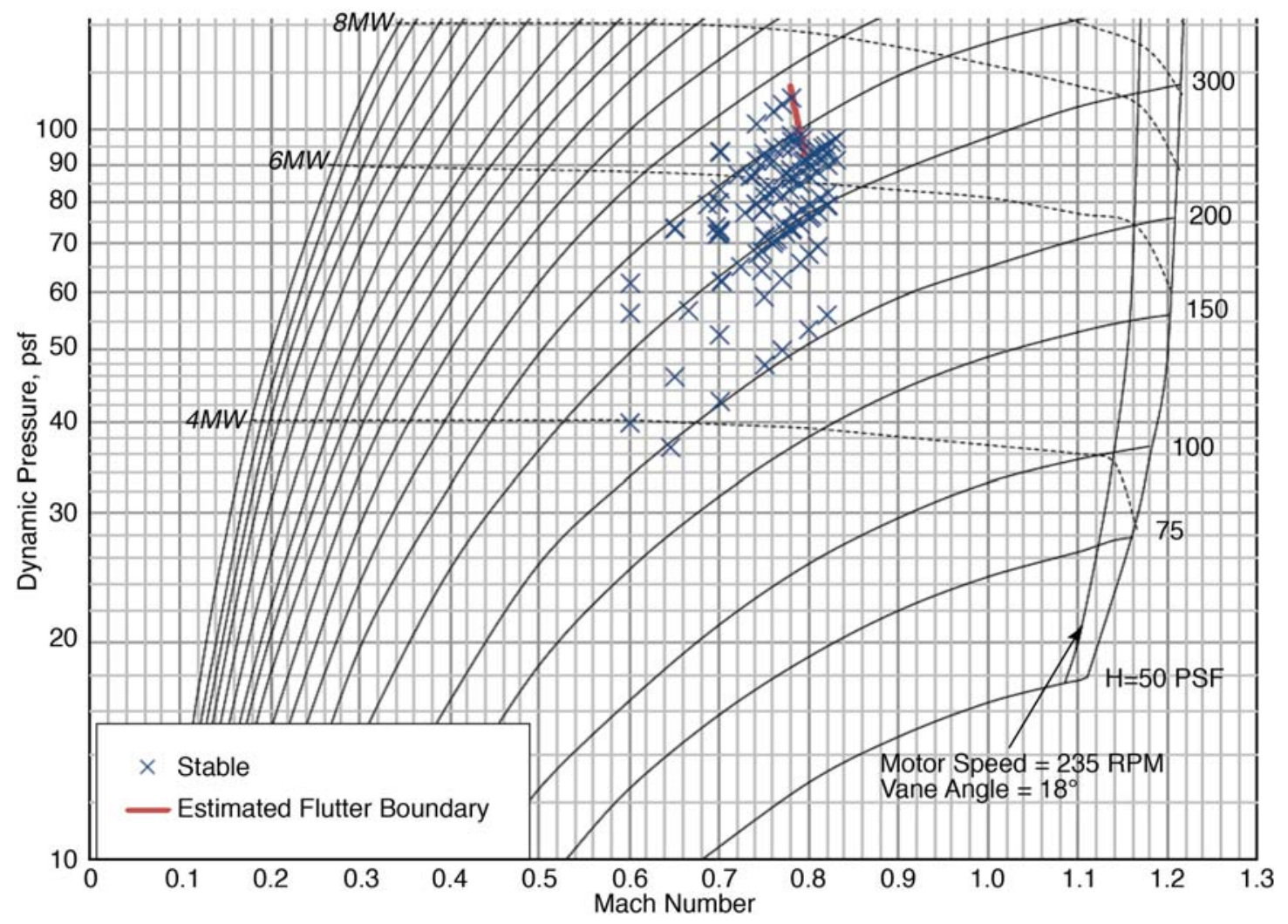

(a) TDT heavy gas boundary with stable points and flutter onset boundary

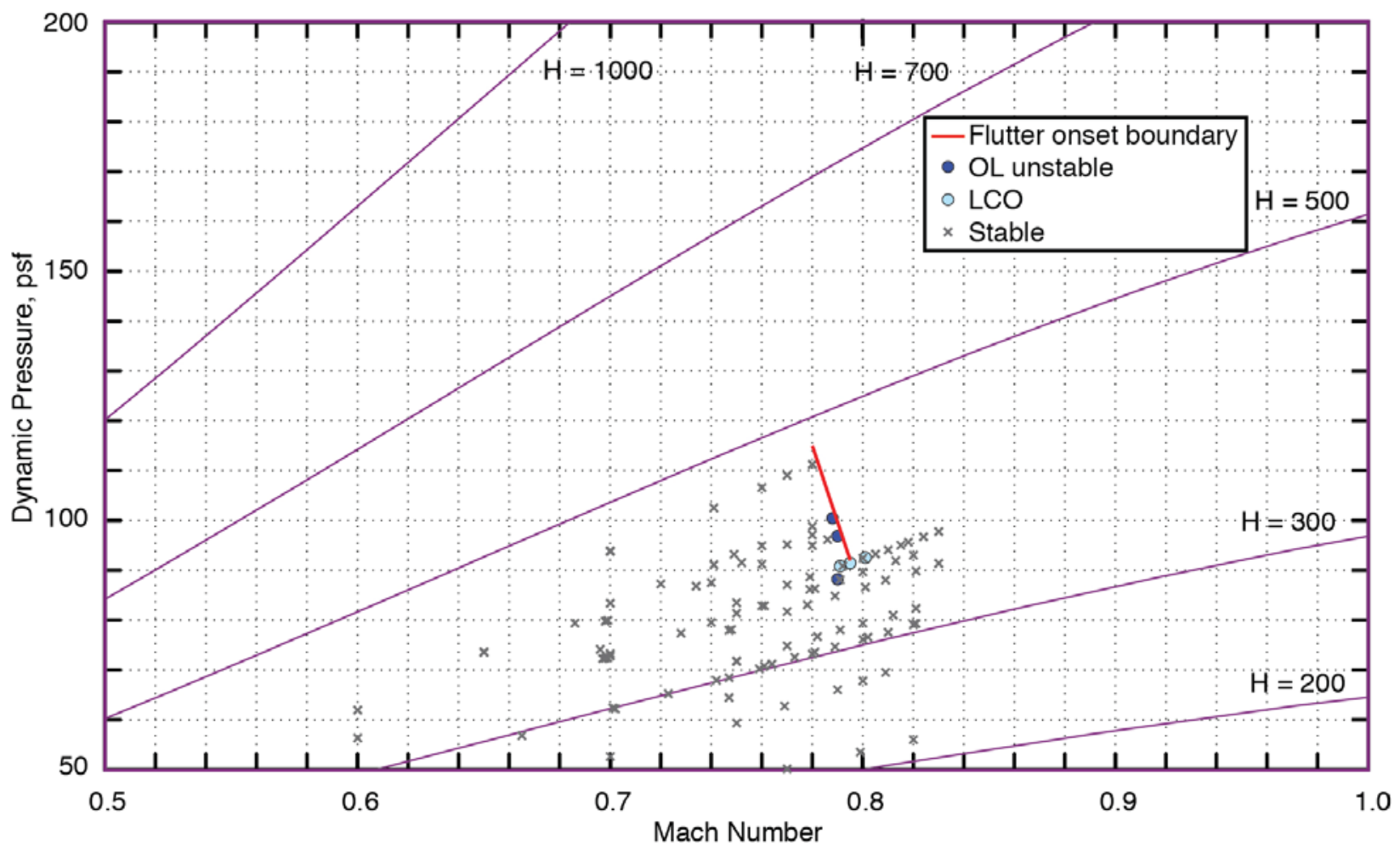

(b) Close-up of onset boundary with LCO and flutter points

Figure 19. Post-holiday flutter onset for AOA $+3^{\circ}$. 


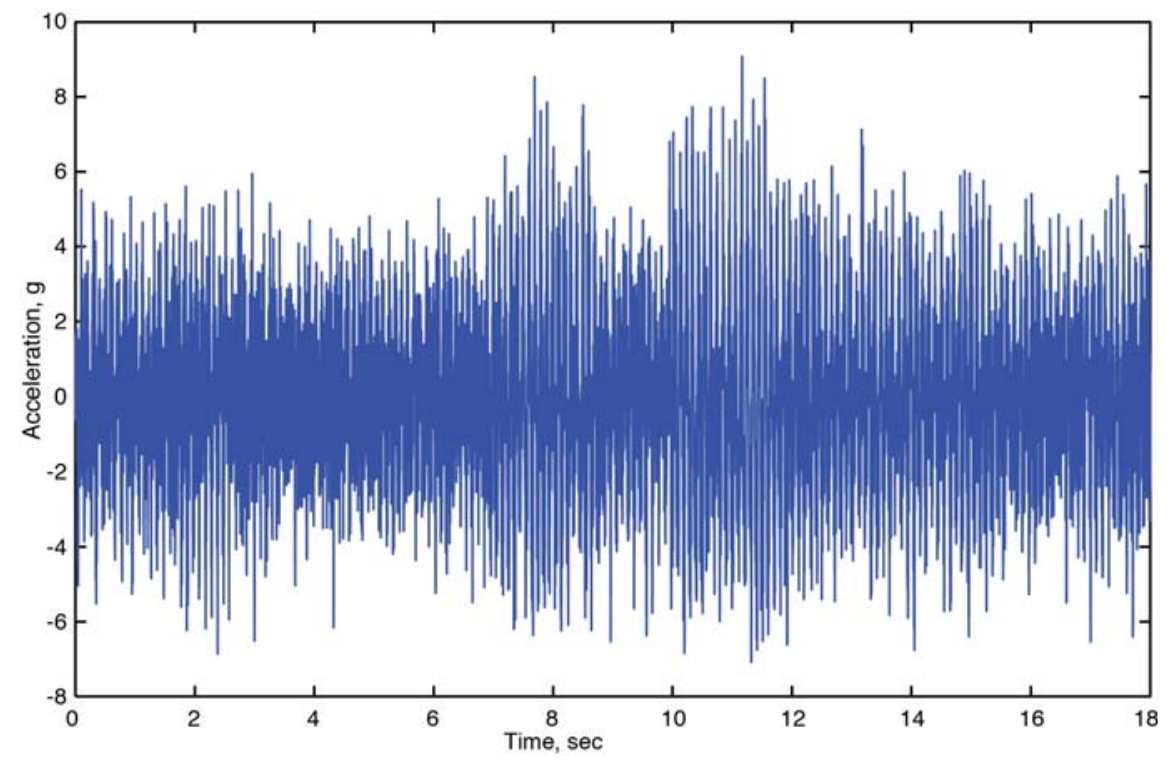

(a) LCO (T644, Run 57, Tab Point 3406, M=0.77 $\overline{\mathrm{q}}=95.43 \mathrm{psf}, \mathrm{AOA}+1^{\circ}$ )

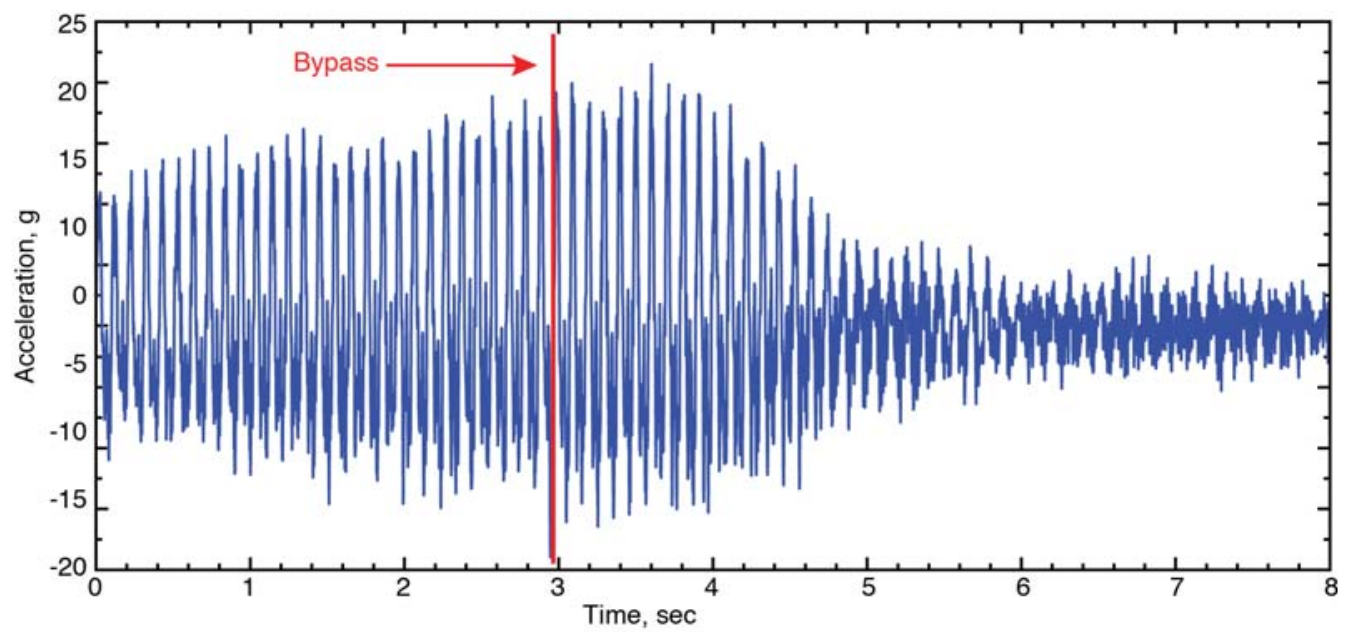

(b) Unstable (T644, Run 57, Tab Point 3407, M=0.78 $\overline{\mathrm{q}}=96.52 \mathrm{psf}$, AOA $+1^{\circ}$ )

Figure 20. Time history data of measured acceleration (ACCZWOTF, see Figure 5) at LCO and unstable tunnel conditions.

As illustrated in the previous flutter data figures, the TBW wind-tunnel model exhibited LCO behavior as well as instabilities that warranted the use of the bypass valves to regain stability. The difference between the points labeled as LCO and those considered to be unstable is illustrated in the time histories presented in Figure 20. The upper time history shows a sustained lower amplitude response with intermittent bursts to higher amplitudes. At this condition the model remains stable while exhibiting periodic characteristically unstable behavior. For all LCO data points, the oscillation frequency observed occurred between $9.2 \mathrm{~Hz}$ and $9.7 \mathrm{~Hz}$ (approximate frequency of second wing bending mode). In the lower part of the figure, an unstable time history is presented. It can be seen that the use of the bypass valves was warranted as the amplitude of acceleration began to diverge. Activating the bypass valves immediately reduced tunnel $\mathrm{M}$ and $\overline{\mathrm{q}}$ and was successful at stabilizing the model following this occurrence of flutter. For all unstable data points, the oscillation frequency observed occurred at approximately $10 \mathrm{~Hz}$. In the previous flutter data figures, for AOAs of $-1^{\circ},+1^{\circ}$, and $+3^{\circ}$, it can also be observed that the LCO behavior occurred at lower H-lines, while the unstable $10 \mathrm{~Hz}$ flutter mechanism was observed at higher $\mathrm{H}$-lines.

Comparison of pre-holiday and post-holiday flutter results is merited due to the inadvertent configuration change previously discussed. Referring back to Table 1, a shift in modal frequencies is seen between pre- 
holiday and post-holiday GVT results. Mode 3 was most impacted by the change with the post-holiday frequency having a $0.5 \mathrm{~Hz}$ decrease. The changes to the modes were also likely accompanied by a change in mode shapes, although mode shapes were not measured experimentally. Figure 21 shows pre- and postholiday flutter onset boundaries for each of the four AOAs. As with the pre-holiday data, the post-holiday data shows the nonlinear effect of AOA/loading on flutter onset. For the lower 3 AOAs, transonic dip occurs at a higher $\mathrm{M}$ and $\overline{\mathrm{q}}$ post-holiday. This is consistent with the greater frequency separation between modes 3 and 4 for the post-holiday model.

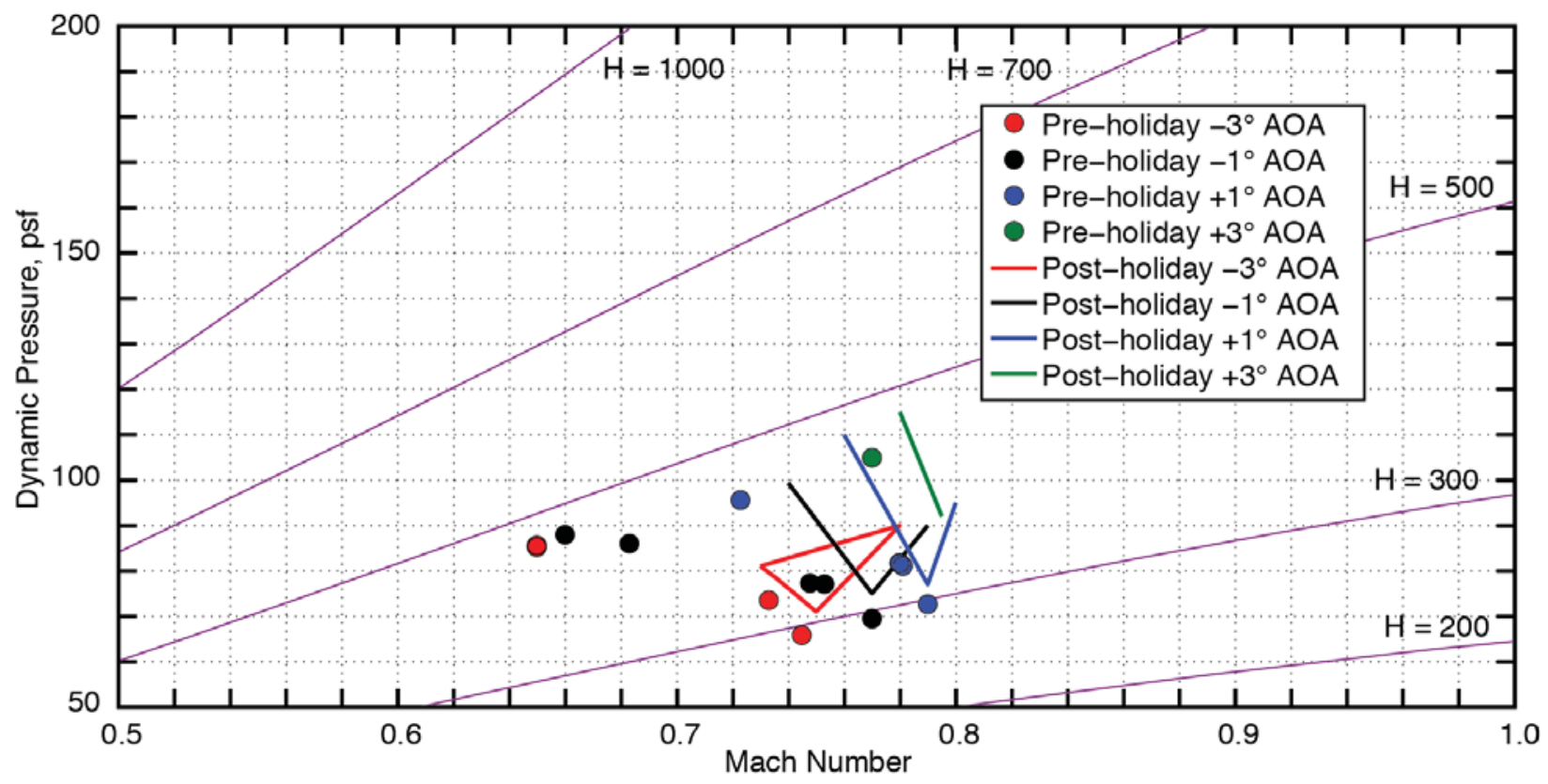

Figure 21. Pre- and post-holiday combined flutter.

During the TBW wind-tunnel test, the bypass valves were used on several occasions in order to regain model stability. Using this method of intervention during flutter allowed the engineers to avoid damage to the model and the tunnel. The valves were generally very effective except on two occasions when the outboard aileron came loose resulting in control surface flutter. This type of flutter involves a coupling between an unbalanced aileron and the first or second bending modes. This flutter mechanism was not intended, and when it occurred the model was well above the control surface flutter onset $\overline{\mathrm{q}}$, and the flutter response persisted for some time before tunnel conditions could be sufficiently reduced. Fortunately, spar pod models are typically very robust and no significant damage resulted from these incidences.

One of the key objectives of the SUGAR Phase II study was to refine the TBW configuration and reduce the uncertainty in the potential benefits, with specific focus on reducing the uncertainty of the wing weight. Experimental validation of the fluter analysis methods used in the design process are a key outcome. Clearly, the open-loop onset boundaries presented so far point to the need to include static nonlinear effects in the flutter analysis. The NASTRAN analysis procedure described in Reference 8 was shown to capture the nonlinear effects associated was static aeroelastic preload. The method was implemented using TBW FEM20 and is described in Reference 2. A comparison of analytical and experimental flutter onset boundaries is shown in Figure 22. The NASTRAN analysis procedure is shown to successfully capture the AOA variation and the minimum flutter $\overline{\mathrm{q}}$. As described in Reference 2, the full-scale design was updated based on these findings, resulting in a small increase in wing weight, and therefore the TBW design still appears to be a viable $\mathrm{N}+3$ configuration. 


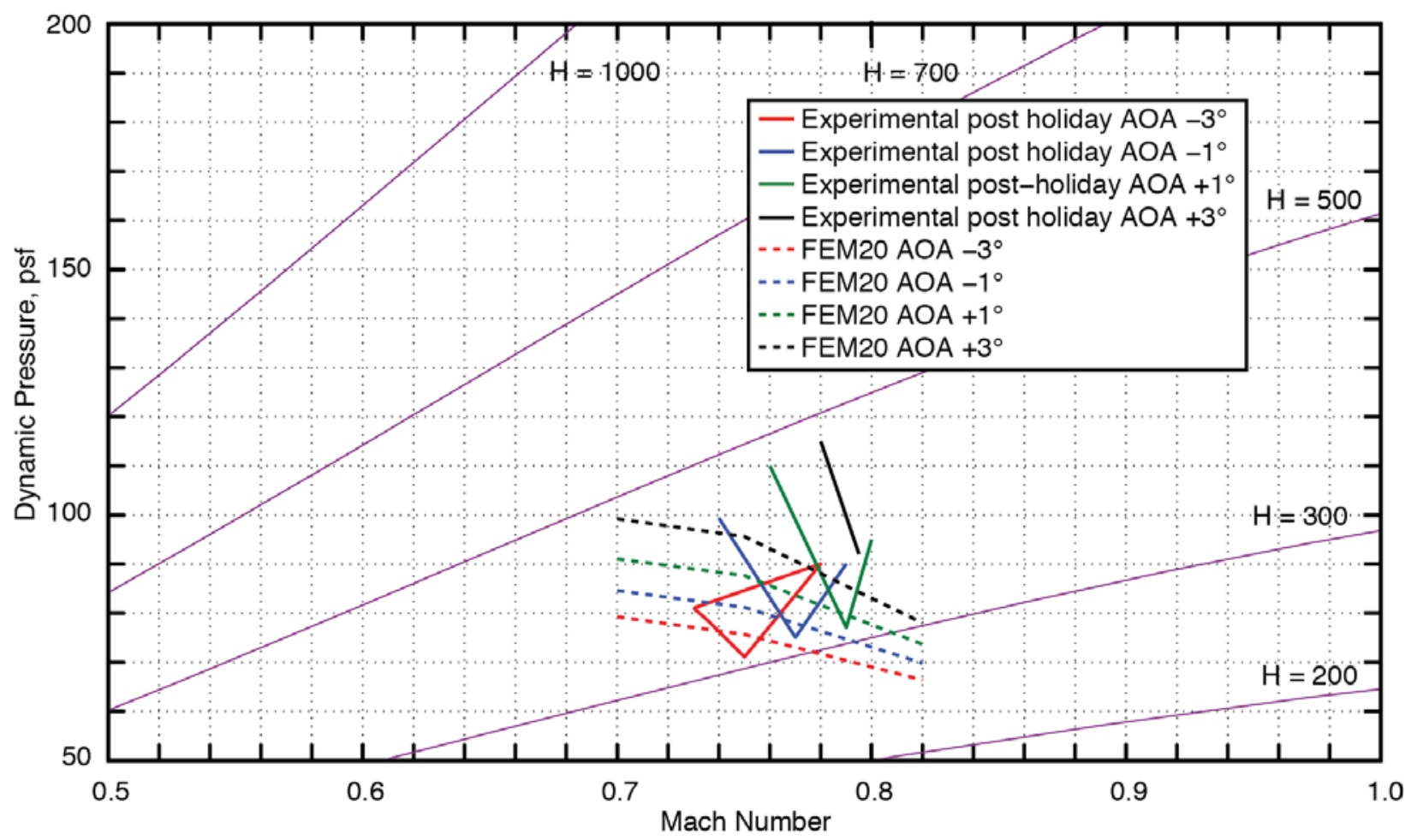

Figure 22. NASTRAN FEM20 flutter analyses and post-holiday flutter onset boundaries for 4 AOAs.

\section{B. Open-Loop System ID}

System identification data were acquired at various open-loop stable M and $\overline{\mathrm{q}}$ combinations. At each tunnel condition, a linear sine sweep was sent to each of the ailerons individually. At some conditions, sweeps were also sent to both surfaces simultaneously for both in- and out-of-phase sweeps. Sweep amplitudes were typically $+/-1.5^{\circ}$ to $+/-3^{\circ}$ with the objective of maximizing signal-to-noise ratios while avoiding excessive model response and actuator saturation.

Figure 23 shows time history data and the associated frequency response function. Here, the sweep is sent to the inboard aileron and the response signal being examined is the wing inboard middle accelerometer (ACCWIMA). Second bending and first torsion mode response can be seen in the accelerometer time history. Response associated with additional bending modes can also be seen in the frequency response function.

The remaining types of open-loop data acquired during the test included control surface dwells and decay, gust response data, and ambient points where the model was responding only to naturally occurring tunnel turbulence. The dwell data were acquired with one of the control surfaces oscillating near the flutter frequency (approximately $10 \mathrm{~Hz}$ ) where the response amplitudes were allowed to stabilize, and then the dwell was stopped while still acquiring time history data. Reference 3 makes extensive use of this data to estimate damping. Open-loop gust response data were acquired at several tunnel conditions, where the AOS vane frequency was varied from $0.5 \mathrm{~Hz}$ to $13.5 \mathrm{~Hz}$ and back to $0.5 \mathrm{~Hz}$ over 120 seconds. Gust response data will be discussed further in the closed-loop section of the paper.

\section{Closed-Loop Testing}

The primary objective of the closed-loop portion of the test was to demonstrate flutter suppression. As discussed previously, two sets of gain scheduled control laws were developed; system ID based and FEM19 based. While both of these control laws were essentially based on the pre-holiday model, they were evaluated using the post-holiday model. As previously discussed, the system ID based controller was based on data acquired at an AOA of $-3^{\circ}$ for two conditions. In contrast, the FEM19 based controllers had more M and $\overline{\mathrm{q}}$ combinations. Also, since the FEM19 controller was based on a linear NASTRAN model, the effects of 

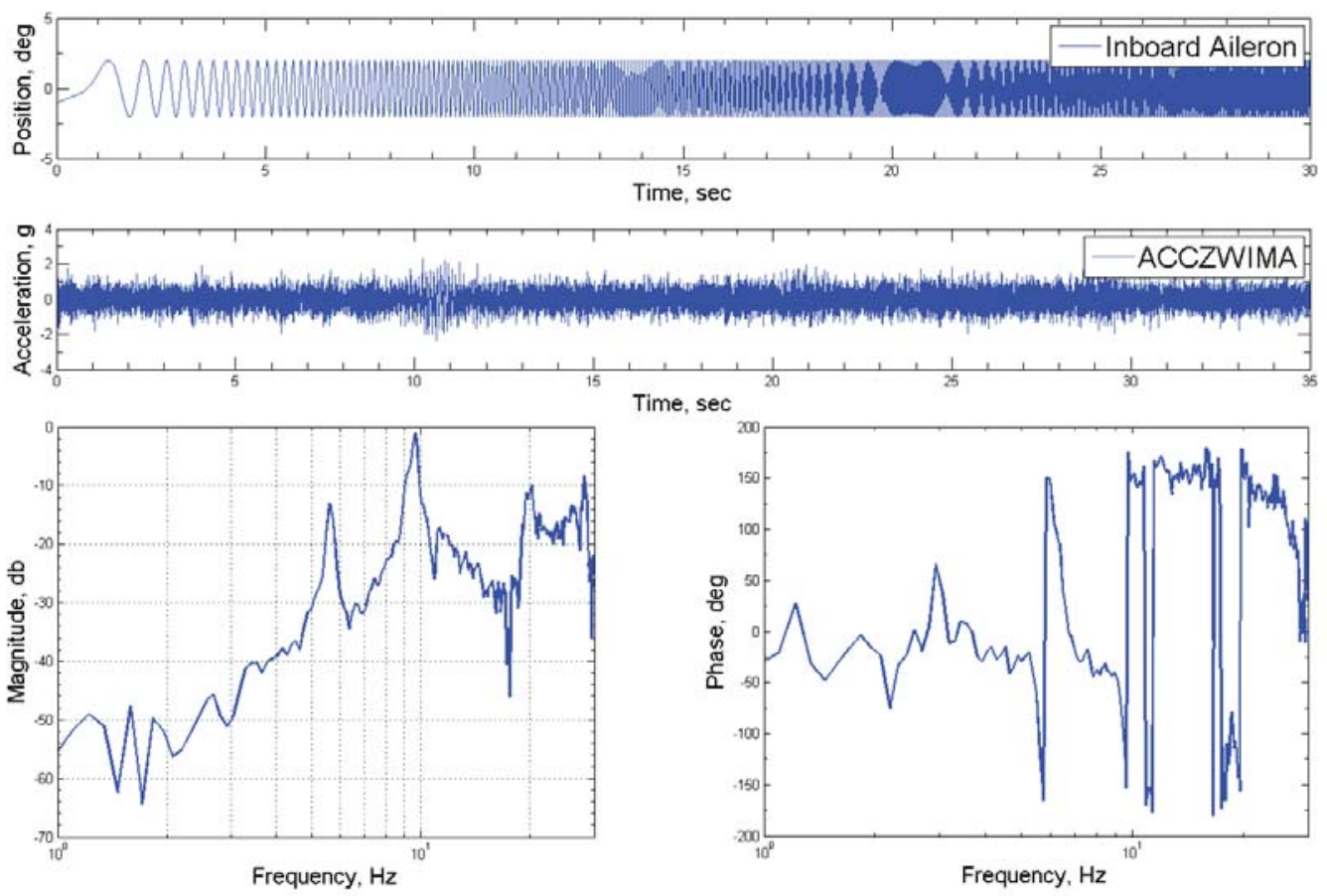

Figure 23. Transfer function estimate of outboard wing accelerometer response to inboard aileron sine sweep (ACCWOJF/Inboard Aileron, Run 13, Point 877, M=0.75, $\overline{\mathrm{q}}=48.9$ psf).

loading and AOA were not included. As will be shown, both control laws suppressed flutter, but the FEM19 controller was used more extensively.

Closed-loop stable points for each control law are plotted along with the previously discussed flutter onset boundaries for the same four AOAs in Figures 24 and 25. Conditions where data were acquired using the SysID based controller are plotted in red, and where the FEM19 based controller was used are plotted in yellow. TDT operations required that $\mathrm{M}$ and $\overline{\mathrm{q}}$ vary along nearly constant $\mathrm{H}$-lines, as previously discussed. Thus, a gap in data along any give H-line indicates that the intervening tunnel conditions were traversed and the model remained stable.

SysID data were only acquired for AOA of $-3^{\circ}$ and $+1^{\circ}$ as shown in Figures 24 and 25, with the most data being acquired at $-3^{\circ}$, as that was the $\mathrm{AOA}$ on which the controller was based. For $-3^{\circ} \mathrm{AOA}$, it is clear that the controller was successful in stabilizing the model through the heart of the flutter onset boundary. At an AOA of $-1^{\circ}$, the SysID controller was also successful in suppressing flutter, but received limited use.

Extensive FEM19 controller data were acquired for all four AOAs. For $-3^{\circ} \mathrm{AOA}$, at least one H-line passed through the heart of the flutter onset region indicating the controller was successful at this AOA. For AOAs of $-1^{\circ}$ and $+1^{\circ}$, the controller was successful in suppressing flutter to a dynamic pressure well above the low points of the transonic dip in the flutter onset boundaries. In the case of $+1^{\circ} \mathrm{AOA}$, testing along the upper of the two H-lines did not proceed to higher $\mathrm{M} / \overline{\mathrm{q}}$ conditions as the control law appeared to be marginally stable. Finally, at $+3^{\circ} \mathrm{AOA}$, only one $\mathrm{H}$-line was tested with flutter suppression demonstrated. Here, higher $\mathrm{M} / \overline{\mathrm{q}}$ conditions were not pursued because of controller stability concerns and the relatively high value of $\bar{q}$ at that point.

While performing closed-loop testing, periodically the loop was opened to assess open-loop stability. Conditions identified as open-loop unstable using this technique were plotted in the open-loop section of the paper. When the loop was opened, it was quickly closed if the model was unstable. Figure 26 shows an example of this technique being used with the FEM19 based controller at an unstable tunnel condition. When the loop was opened, response grew rapidly until the loop was closed. 


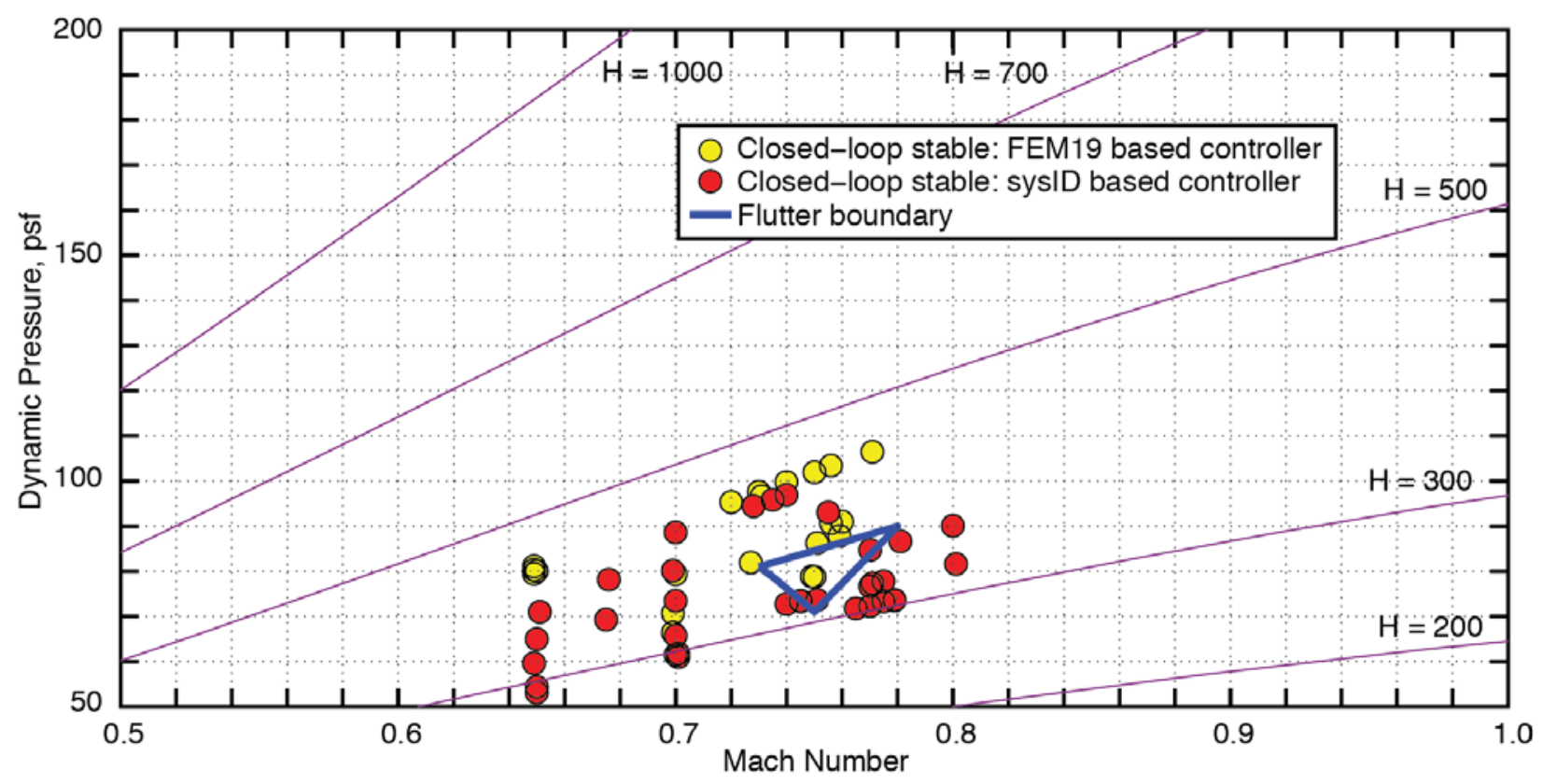

(a) $\mathrm{AOA}=-3^{\circ}$

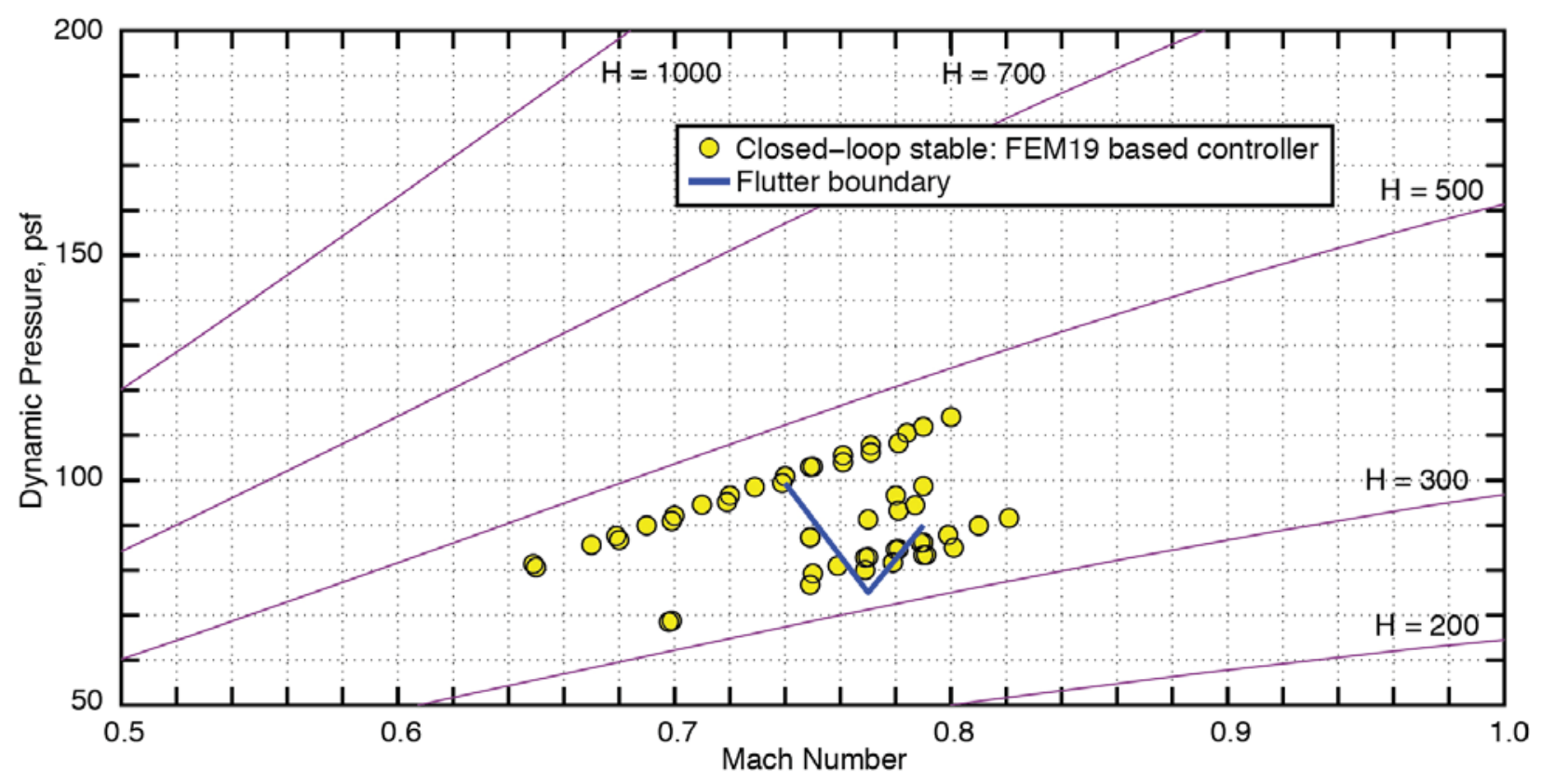

(b) $\mathrm{AOA}=-1^{\circ}$

Figure 24. TDT experimental flutter and closed-loop stable points at negative AOAs. 


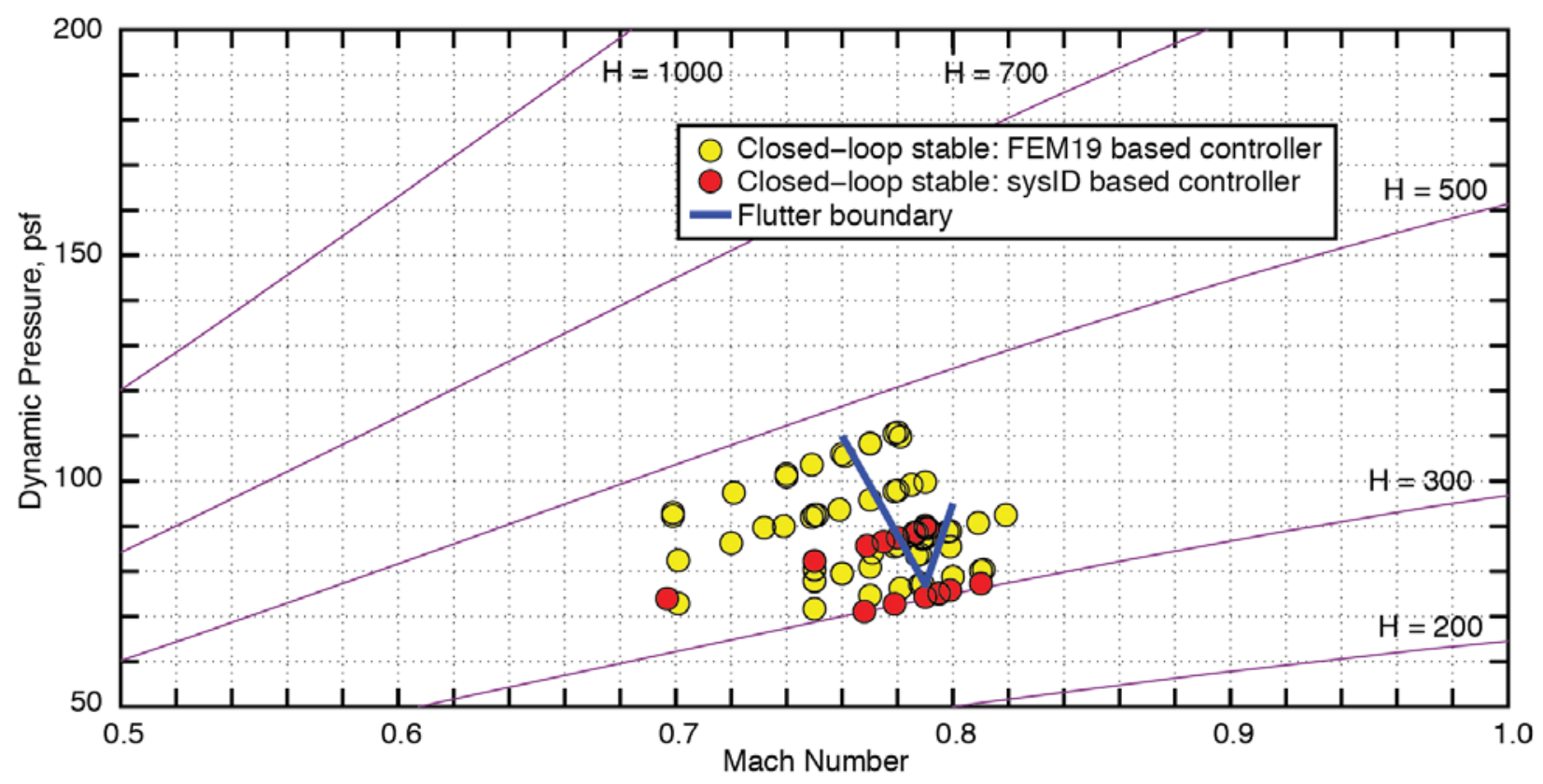

(a) $\mathrm{AOA}=+1^{\circ}$

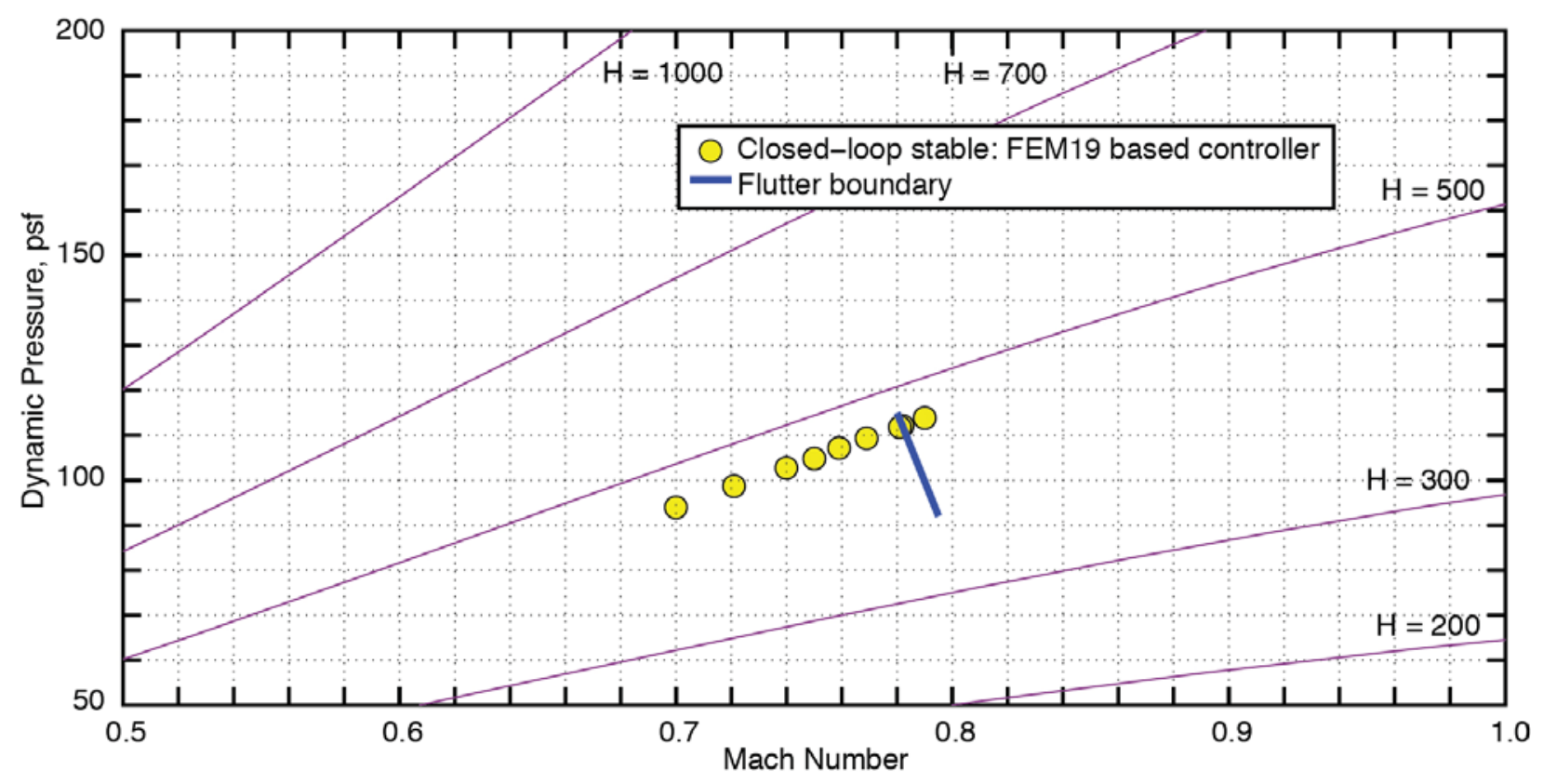

(b) $\mathrm{AOA}=+3^{\circ}$

Figure 25. TDT experimental flutter and closed-loop stable points at positive AOAs. 


\section{Closed-Loop System ID and Gust Response Testing}

Closed-loop system identification attempts were made but were not very successful. Control surface sweeps were conducted as they were with open-loop procedures, where a fixed amplitude sweep from 0 to $30 \mathrm{~Hz}$ was run to acquire the data. The sweeps were executed in two different configurations; inboard and outboard ailerons individually excited, and inboard and outboard ailerons simultaneously excited (both in-phase and out-of-phase with each other). However, the response due to the control surface sweeps for the closed-loop points in the open-loop unstable region was lost in the overall noise and was insufficient for system ID.

Gust load alleviation control laws were not specifically designed for this test. However, the effect of the flutter suppression controllers on gust response could be assessed. The procedure here was to acquire two gust sweeps; one open-loop and one closed-loop. This allowed open- and closed-loop data to be compared at identical tunnel conditions. Figure 27 shows an example for the FEM19 based controller at an AOA of $+1^{\circ}$. Here, the transfer function between a sine wave fitted to the gust input and model response is estimated with and without the loop closed. As the controller was designed to suppress a $10 \mathrm{~Hz}$ flutter mode, it is not surprising to see the most significant response between the second bending mode frequency, around $9.6 \mathrm{~Hz}$, and $10 \mathrm{~Hz}$.
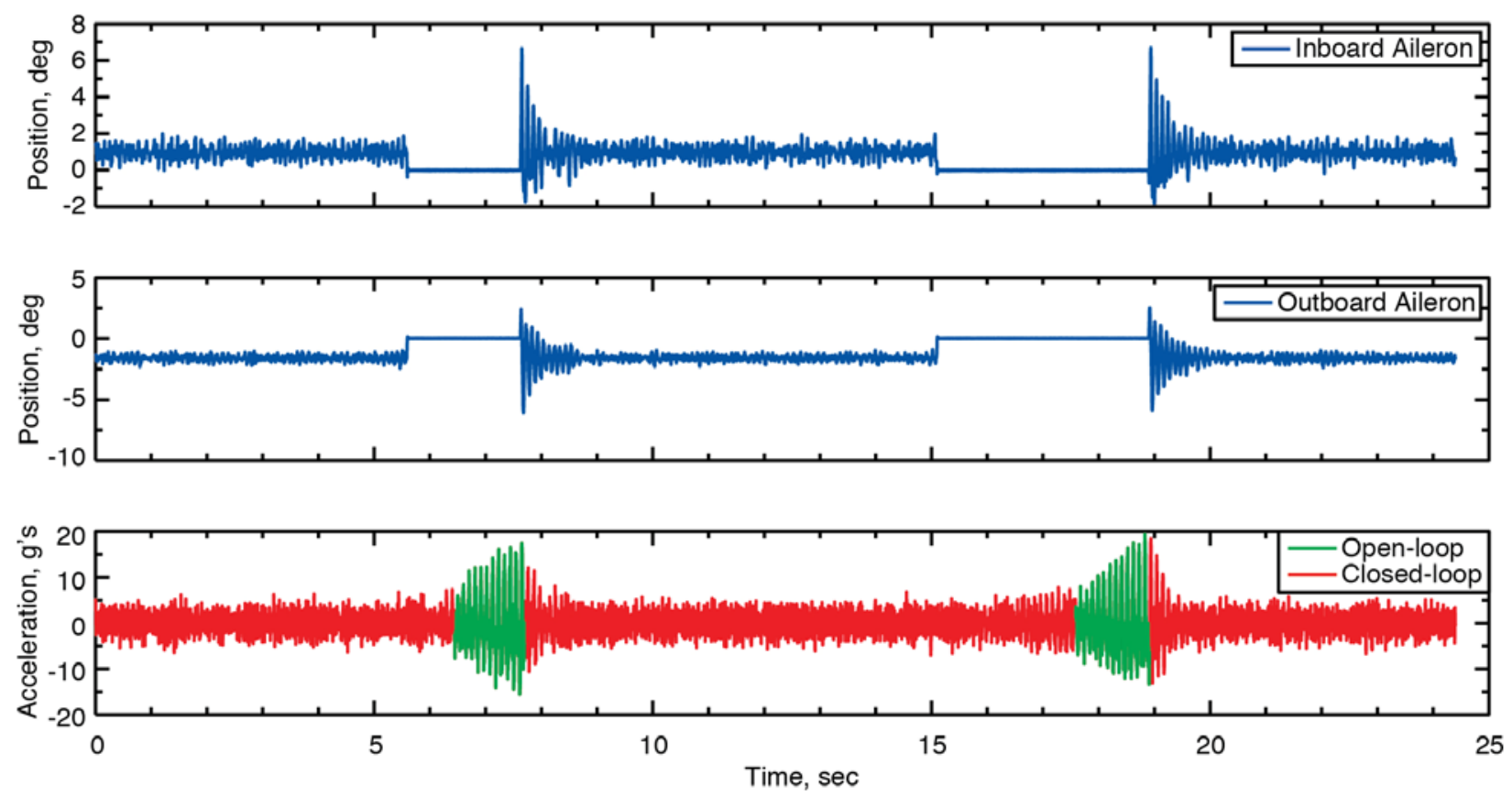

Figure 26. FEM19 controller: open-loop/closed-loop demonstration (T644, Run 56, Tab Point 3228, M=0.79 $\overline{\mathrm{q}}=87.3$ psf, Signal: ACCZWOTF, See Figure 5).

\section{Concluding Remarks}

In April 2014, Boeing and NASA Langley Research Center successfully completed the wind-tunnel test of an aeroelastically scaled semispan wind-tunnel model of a truss-braced wing concept. The primary goals of the test were to identify the open-loop flutter boundary and then demonstrate flutter suppression. A secondary goal was to demonstrate gust load alleviation. This paper has provided an overview of the TBW wind-tunnel model, which was a $15 \%$ scaled version of a Boeing $\mathrm{N}+3$ vehicle concept. Descriptions of the hardware including the wind tunnel, the wind-tunnel model, and digital control systems were provided. Open-loop flutter results for the TBW wind-tunnel model have been discussed, and as predicted by linear analysis, the flutter mechanism was a coalescence of modes 3 and 4 at approximately $10 \mathrm{~Hz}$. The flutter onset boundaries showed a significant variation with AOA. This nonlinear variation appears to be a characteristic of the TBW configuration. These findings were used to update the design process for the full scale TBW vehicle, and the design concept remains viable. Some experimental LCO conditions were also identified, 
especially for $-3^{\circ}$ AOA. Flutter suppression was successfully demonstrated for control laws designed using ASE models derived from both experimental data and from finite element models. Although the control laws were developed for flutter suppression, some gust load alleviation was demonstrated between 6 and 10 $\mathrm{Hz}$.
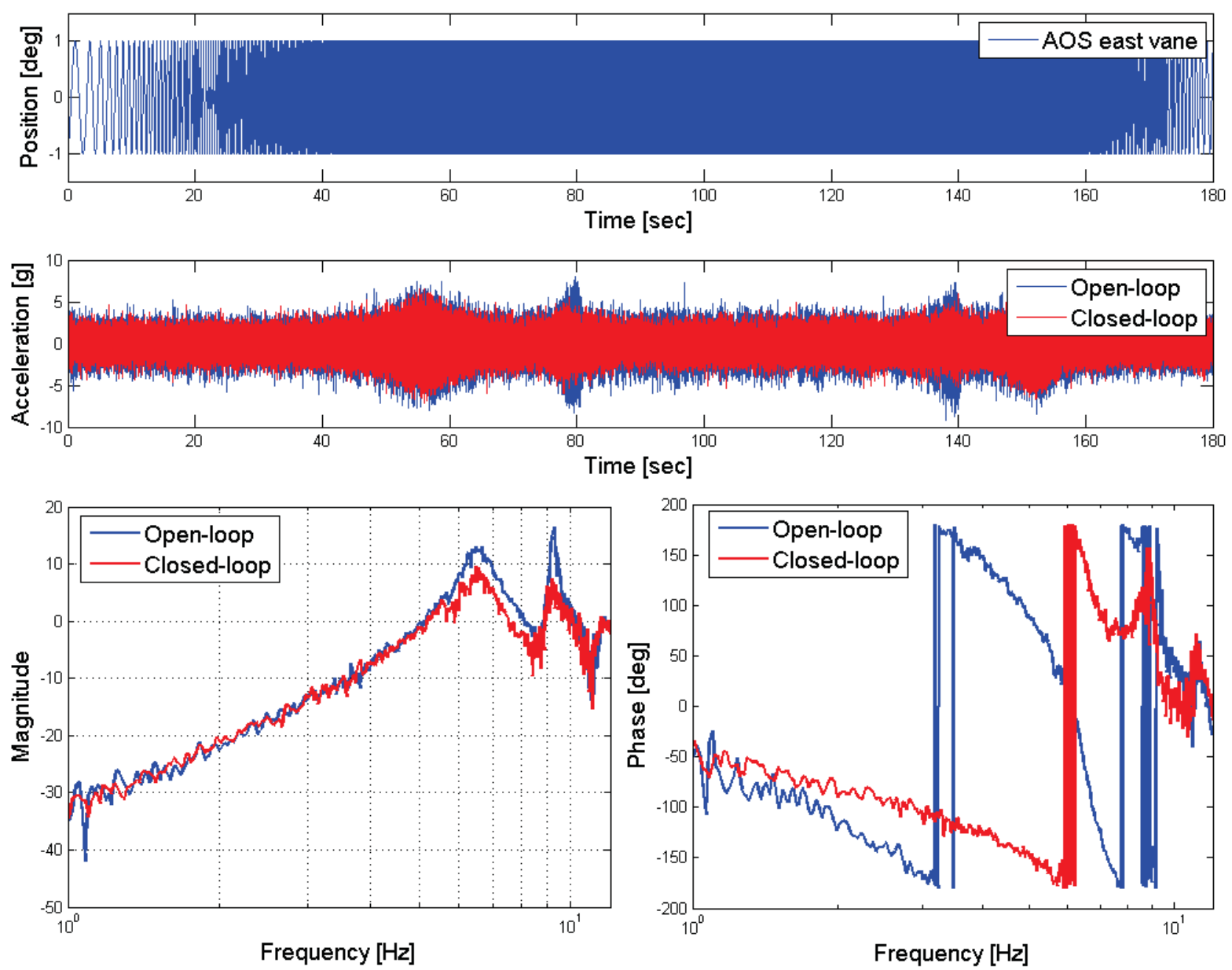

Figure 27. Run 59, Tab 3705 (OL), Tab 3707 (CL). M=0.75 $\bar{q}=64$ psf, AOA $+1^{\circ}$, Signal: ACCZWOTA, See Figure 5.

\section{References}

${ }^{1}$ Bradley, M. and Droney, C., "Subsonic Ultra Green Aircraft Research: Phase I Final Report," NASA CR-2011-216847, April 2011.

${ }^{2}$ Allen, T., "SUGAR Truss Braced Wing Full Scale Aeroelastic Analysis and Dynamically Scaled Wind Tunnel Model Development," Proceedings of the 55th AIAA/ASME/ASCE/AHS/ASC Structures, Structural Dynamics, and Materials Conference, Kissimmee Florida, Jan. 2015.

${ }^{3}$ Bradley, M., Droney, C., and Allen, T., "Subsonic Ultra Green Aircraft Research: Phase II Final Report," Contractor Report NNL08AA16B, May 2014.

${ }^{4}$ Perry, B., Noll, T., and Scott, R., "Contributions of the Transonic Dynamics Tunnel to the Testing of Active Control of Aeroelastic Response," AIAA Dynamics Specialists Conference, No. 1999-6108, Atlanta Georgia, April 2000.

${ }^{5}$ Scott, R., Castelluccio, M., Coulson, D., and Heeg, J., "Aeroservoelastic Testing of Free Flying Wind Tunnel Models Part 2: A Centerline Supported Fullspan Model Tested for Gust Load Alleviation," NASA TP 2013-218051, Feb. 2014.

${ }^{6}$ Scott, R. C., Vetter, T. K., Penning, K., Coulson, D. A., and Heeg, J., "Aeroservoelastic Testing of Free Flying Wind Tunnel Models Part 1: A Sidewall Supported Semispan Model Tested for Gust Load Alleviation and Flutter Suppression," NASA TP 2013-218051, Oct. 2013. 
${ }^{7}$ Silva, W., Perry, B., Florance, J., Sanetrik, M., Wieseman, C., Stevens, W., Funk, C., Hur, J., Christhilf, D., and Coulson, D., "An Overview of the Semi-Span Super-Sonic Transport $\left(\mathrm{S}^{4} \mathrm{~T}\right)$ Wind-Tunnel Model Program," Proceedings of the 53rd AIAA/ASME/ASCE/AHS/ASC Structures, Structural Dynamics and Materials Conference, Honolulu Hawaii, April 2012.

${ }^{8}$ Coggin, J., Kapania, R., and et. al., "Nonlinear Aeroelastic Analysis of a Truss Braced Wing Aircraft," Proceedings of the 55th AIAA/ASME/ASCE/AHS/ASC Structures, Structural Dynamics, and Materials Conference, No. 2104-0335, National Harbor, MD, Jan. 2014.

${ }^{9}$ Bartels, R. E., Scott, R. C., Allen, T., Sexton, B., and Funk, C., "Computed and Experimental Flutter/LCO Onset for the Boeing Truss-Braced Wing Wind-Tunnel Model," Proceedings of the 32nd AIAA Applied Aerodynamics Conference, No. 2014-2446, Atlanta, GA, June 2014. 1969.

${ }^{10}$ Staff of the Aeroelasticity Branch, "The Langley Transonic Dynamics Tunnel," Langley Working Paper LWP-799, Sept.

${ }^{11}$ Corliss, J. M. and Cole, S. R., "Heavy Gas Conversion of the NASA Langley Transonic Dynamics Tunnel," Proceedings of the 20th Advanced Measurements and Ground Testing Technology Conference, No. 98-2710, Albuquerque, NM, June 1998.

${ }^{12}$ Cole, S. R. and Rivera Jr, J. A., "The New Heavy Gas Testing Capability in the NASA Langley Transonic Dynamics Tunnel," Royal Aeronautical Society Wind Tunnels and Wind Tunnel Test Techniques Forum, No. 4, Cambridge, UK, April 1997.

${ }^{13}$ Reichenbach, E., Castelluccio, M., Sexton, B., and Scott, R., "Aerodynamic Efficiency Improvement Final Report Volume 2: Aeroservoelastic Test Program," AFRL Contractor Report FA8650-05-C-3500, Boeing Research and Technology Computational Sciences, 2010.

28 of 28 